\title{
Second-order Accurate Interface- and Discontinuity-aware Diffusion Solvers in Two and Three Dimensions
}

\author{
William W. Dai and A.J. Scannapieco \\ Los Alamos National Laboratory \\ Corresponding author: William W. Dai, email: dai@lanl.gov \\ phone: (505)665-1967, fax: (505)665-4972
}

Revised April 15, 2014

\begin{abstract}
A numerical scheme is developed for two- and three-dimensional time-dependent diffusion equations in numerical simulations involving mixed cells. The focus of the development is on the formulations for both transient and steady states, the property for large time steps, second order accuracy in both space and time, the correct treatment of the discontinuity in material properties, and the handling of mixed cells. For a mixed cell, interfaces between materials are reconstructed within the cell so that each of resulting sub-cells contains only one material and the material properties of each sub-cell are known. Diffusion equations are solved on the resulting polyhedral mesh even if the original mesh is structured. The discontinuity of material properties between different materials is correctly treated based on governing physics principles. The treatment is exact for arbitrarily strong discontinuity. The formula for effective diffusion coefficients across interfaces between materials are derived for general polyhedral meshes. The scheme is general in two and three dimensions. Since the scheme to be developed in this paper is intended for multi-physics code with adaptive mesh refinement (AMR), we present the scheme on mesh generated from AMR. The correctness and features of the scheme are demonstrated for transient problems and steady states in one-, two-, and three-dimensional simulations for heat conduction and radiation heat transfer. The test problems involve dramatically different materials.
\end{abstract}

Keywords: radiation, difusion, mixing, interface, implicit

\section{Introduction}

In multi-physics simulations, for example, hydrodynamics and radiation diffusion, mixed cells are often generated no matter how fine the resolution of a simulation is. In many simulations there is more than one material in one cell, such as melting metal and gas. Mesh refinement only can reduce the number of materials within a cell, but cannot eliminate mixed cells in simulations. Treatment of mixed cells is critical for many applications, but material property of mixture of materials is often unknown.

Numerical techniques for diffusion equations $[1,2,3,6]$ may be divided into explicit and implicit methods. An explicit scheme, for example, the forward Euler method, is simple, but the size of time 
step is limited by a stability condition that is normally much smaller than the required accuracy of physics problems. On the other hand, in implicit methods the size of time step is not limited by numerical stability conditions, and therefore it may be changed according to the requirement of physics problems.

Two typical implicit methods are the backward Euler method and Crank-Nicolson method. The backward Euler method is first order accurate in time, but numerical errors in the method undergo quick damping for large time steps. Therefore the method is very useful for large time steps and steady states. Although Crank-Nicolson method is second order accurate, numerical errors do not damp out for large time steps, and significant numerical errors will be introduced when time steps are large. This is the reason Crank-Nicolson method cannot be used for steady states. The formulation for steady states is very important for problems involving dramatically different materials even for transient problems. A given time step may be considered so large for some material that the formulation for steady state is more appropriate for the material than the formulation with the second order of accuracy, but the time step is so small for some other material that accuracy in time is more important, as illustrated in Fig.1 in which there are three materials. The left image in the figure is the diffusion coefficients of the three materials, and right image is temperature of each material. If we are not interested in how temperature changes with time in the material in the thin layer, we can give a very large time step relative to the material in the thin layer.

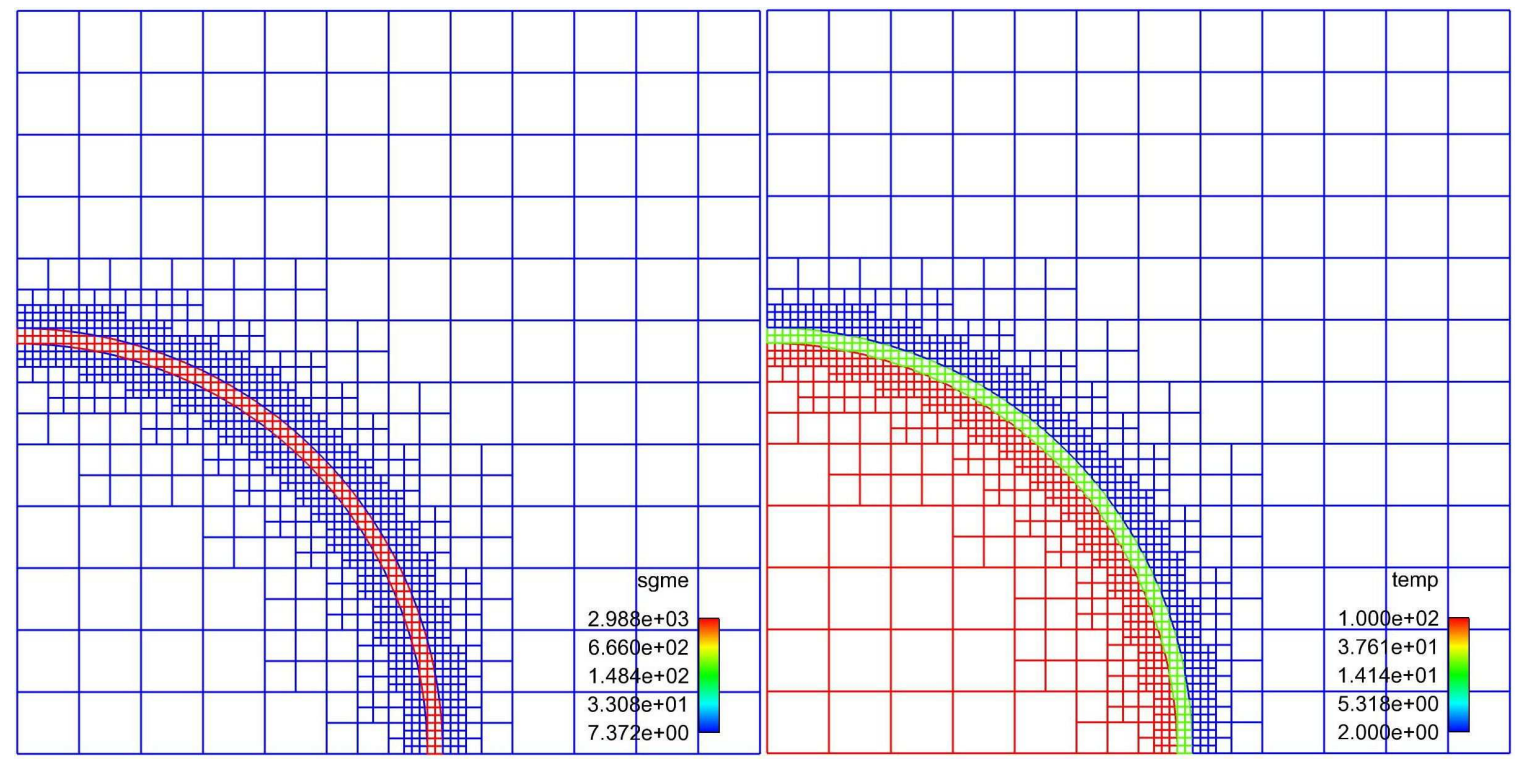

Figure 1: The heat conduction coefficients of three materials (left image) and the initial temperature in a mesh (right image).

For systems of multi-materials with dramatically different material properties, the correct treatment for the discontinuity of material properties is important. A typical approach for the flux calculation near material interfaces on unstructured meshes is to use mathematical approximations, for example, Taylor expansion or some weighted average of two adjacent materials, but not governing physics laws. This approach would introduce numerical errors when thermal properties of two materials are very different.

There are many investigations on numerical methods for diffusion equations $[4,8,9,11-15,17$ - 
$22,24,25,27,28,30-33,35]$. Some work on unstructured meshes but is only first order accurate in time. Some are second order accurate but can not give correct steady states for large time steps. Some work well for clean cells (i.e., cells with single material), but do not deal with mixed cells. Some are designed for mixed cells, but lack special consideration for the discontinuity at material interfaces. In this paper, we will develop a numerical method for two- and three-dimensional diffusion equations for systems of multi-materials with arbitrarily different thermal properties. We will focus on the case in which there are mixed cells, although the resulting scheme is perfectly appropriate for general polyhedral meshes and structured meshes with and without adaptive mesh refinement (AMR). We will give formula for effective diffusion coefficient across a material interface for flux calculations on polyhedral meshes. The scheme is formally second order accurate in both space and time, works for any size of time steps, and will give exact steady states when time steps are very large.

Although AMR, implicit diffusion, and the treatment of mixed cells increase the complexity of numerical algorithms, AMR has gradually become common practice in many institutions. Even with AMR and implicit treatment of diffusion, it is still a challenge to effectively simulate some physics problems through Euelrian methods. Recently, in these institutions efforts are put on interface reconstruction for hydrodynamics and diffusion. The sub-cell structure within mixed cells is considered necessary for Eulerian methods to solve some problems, particularly those problems that are traditionally solved through Lagrangian methods. In many institutions, the sub-cell structure becomes increasingly important, for example see presentations in 2013 Multi-material Hydrodynamics Conference [36,37]. The work reported in this paper is one of the efforts, which was reported in the conference.

The plan of the papers is as follows. The second section is for the basic equation to be solved. In the third section we will describe mixed cells through volume fraction of each material in cells. A procedure to reconstruct interfaces between materials within a numerical cell is presented there. In the fourth section we will present our numerical scheme, which includes the simultaneous discretization in space and time, realization of the second order of accuracy in time, consideration of large time steps and steady states, and correct treatment for the discontinuity of material properties. The scheme may apply to two-, and three-dimensional general polyhedral meshes. After that is the section for numerical examples, which include one-, two-, and three- dimensional problems involving more than one material with and without mixed cells. The last section is the conclusion of this paper and discussion for future work.

\section{Diffusion Equations}

The diffusion equation for temperature $T(t, \mathbf{r})$ is

$$
\frac{\partial u(T)}{\partial t}+\nabla \cdot \mathbf{F}(T)=S(T) .
$$

Here $\mathrm{u}(\mathrm{T})$ is energy density, a function of temperature, and $\mathbf{F}$ is energy (or heat) flux, defined as

$$
\mathbf{F} \equiv-\kappa \nabla T .
$$

The coefficient $\kappa$ represents diffusion coefficient, and it may be very different for different materials.

$S$ is a source term. Examples of the diffusion equations include heat conduction and radiation diffusion, in which

$$
u(T)=C_{v} T+a T^{4}
$$




$$
\mathbf{F}=-\left(\kappa_{0} \nabla T+\sigma \nabla T^{4}\right)
$$

Here $C_{v}$ is the heat capacity of material, and $C_{v} \equiv \rho c_{v}$ with $\rho$ being material density and $c_{v}$ specific heat capacity, $a$ is the radiation constant, and $\kappa_{0}$ and $\sigma$ are diffusion coefficients of material and radiation. In Eqs.(4), temperature equilibrium is assumed between materials and radiation.

In general, Eq.(1) is a nonlinear equations, and the function $u(T)$, the coefficients, $\kappa, \kappa_{0}$, $C_{v}$, and $\rho$ may be dramatically different for different materials involved in one simulation. The nonlinearity and discontinuity of material properties are two difficulties in numerical simulations.

\section{Material Mixing and Interface Reconstruction}

The numerical method to be developed here is intended to be used in a code for multi-physics [34], including hydrodynamics, magnetohydrodynamics, material strength, heat conduction, and radiation temperature diffusion. It is an extension of the work reported in [12] to include mixed cells and unstructured meshes. Typically, there are mixed cells involved in the applications of the code. Figure 1 shows a mesh with three materials, and there are mixed cells between materials.
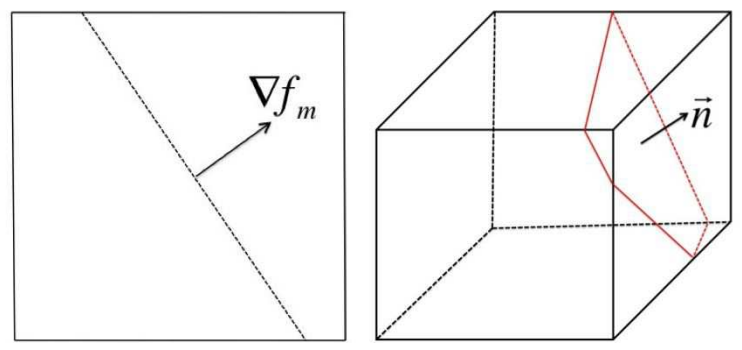

Figure 2: Illustration of normal direction of reconstructed material interface in two (left) and and three (right) dimensions.

For some problems, materials within a mixed cell are in a state of thermal non-equilibrium. Accurate estimate of the temperature of each material within the cell is very important for certain subsequent physics, for example, nuclear reaction rates. The subsequent physics calculations may sensitively depend on the specific temperature of some material in the mixed cell, but not a homogenized temperature. Different temperatures may results in very different outcomes. For this reason, we could not homogenize the temperatures of materials within a mixed cell. Also, tables of equation of state (EOS) for real materials are very important for many problems. Although EOS tables of pure material are available, tables of mixture of material are not. Incorrect use of EOS tables will often result in incorrect temperature of some specific material, which again would change the calculation of subsequent physics packages.

AMR would dramatically reduce the total volume of mixed cells and help resolving material interfaces since we always refine any mixed cell to the finest level. No matter how fine the mesh is, there always exist mixed cells. Theoretically, after reaching a certain resolution for a given problem, the contributions of those mixed cells could be very small compared to overall solution of pure cells. Therefore, after that resolution, the contribution from mixed cells may be negligible. But, we are far away from that resolution in daily simulations even in two dimensions. At least before we reach that resolution, further improvement in numerical techniques is needed to better resolve sub-grid physics. 
Typical interface reconstruction in numerical simulations is through a single line in two-dimension or a single plane in three dimensions within a mixed cell with two materials. This kind of reconstruction is called linear interface reconstruction. The location of an interface is uniquely determined by the normal direction of the interface for the given volume fractions of each material within the cell. Although there are several approaches for linear interface reconstruction [10,23,26,29], we use the gradient-based method. Most of existing papers about interface reconstruction are only for the two-dimensional case. Many papers deal with only two materials. In this paper, we consider the interface reconstruction with any number of materials in both two- and three-dimensional meshes with AMR.

Within the context of this paper, the generation of mixed cells is mainly due to two mechanisms. The first one is from initial condition. Many problems involve circular initial distribution of material. Since we use rectangular cells to simulate circular problems, some cells near interfaces between materials would have more than one material, i.e., those are mixed cells. Even if there are no mixed cells initially in simulations, advection in multi-material hydrodynamics in Eulerian grids often results in mixed cells, and remapping in arbitrary Lagrangian-Eulerian (ALE) simulations will also generate mixed cells. Therefore, mixed cells are often in numerical simulations.

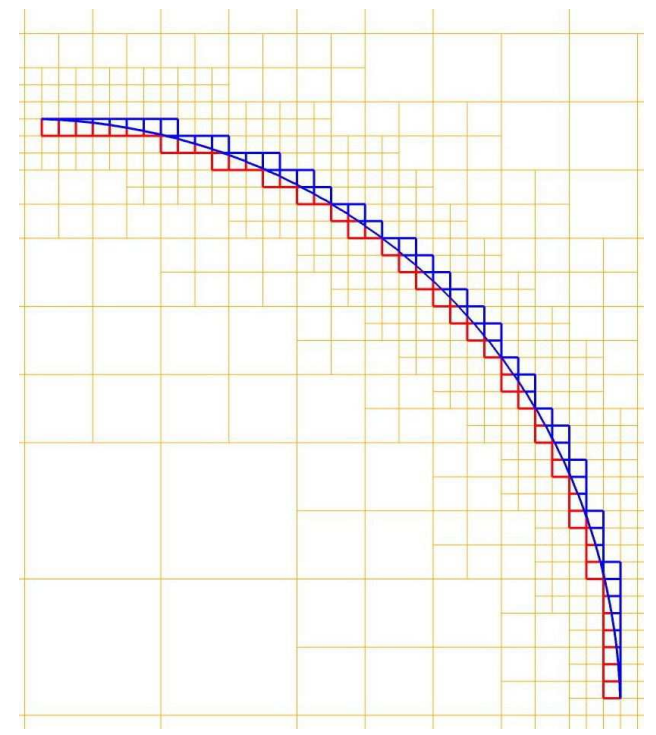

Figure 3: Reconstructed material interfaces within mixed cells in two dimensions.

We first consider a simple situation in which any mixed cell contains only two materials. For a given mesh and volume fraction $f$ of one material in each cell, we first approximately obtain the gradient of the volume fraction, $\nabla f$, on each cell. This calculation typically involves neighboring cells of the cell. The direction of the gradient $\nabla f$ is shown in Fig.2 in two and three dimensions. After obtaining the direction of the gradient, we draw a line or plane within the cell perpendicular to the gradient, and then we move the line or plane along the direction of gradient until the two volumes separated by the line within the cell match the volumes of the two materials in the cell. Since the interfaces are constructed cell by cell, there is typically a gap between two interfaces of two adjacent cells. Figure 3 displays the interfaces in a two-dimensional mesh. We will solve diffusion equations on the resulting unstructured mesh, which includes arbitrary polygons or polyhedrons. Fig.4 shows sub-cells and reconstructed interfaces in a three-dimensional mesh, which include arbitrary polyhedrons. In Fig.4 we show only mixed cells, and each color in the figure 
represents one material.

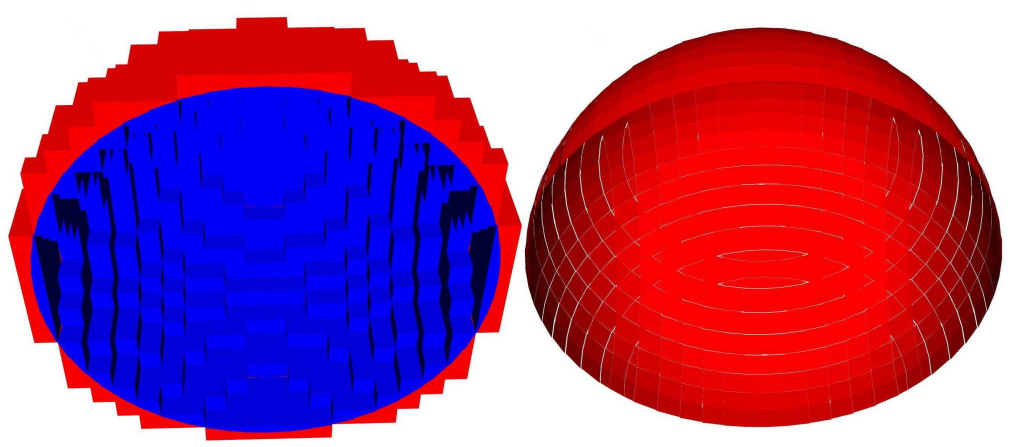

Figure 4: Unstructured cells resulting from reconstruction of material interface within threedimensional mixed cells (left) and the reconstructed material interfaces between two materials.

We next consider the problems in which some mixed cells have more than two materials. For this case, we make a significant assumption that the interfaces between the materials within a mixed cell are parallel to each other. This assumption has been used in production codes of hydrodynamics for years. This assumption significantly simplifies the calculation of interfaces, but may limit applications. From this assumption, each mixed cell has only one direction normal to all the interfaces within the cell.

To determine the normal direction to the interfaces of each mixed cell, we first calculate the gradient of each material, $\nabla f_{m}$, and then we construct a special function of material $q(m)$, which is defined

$$
q(m) \equiv\left|\nabla f_{m}\right|^{2} \sqrt{f_{m}}
$$

The gradient of the material that has the largest value of $q(m)$, supposing $m_{0}$, will be considered as the normal direction of all the interfaces within the cell. After that, we have to determine the order of materials along the normal direction within the cell. For the order, we define another function $p(m)$ defined as

$$
p(m) \equiv \mathbf{n}(m) \cdot \mathbf{n}\left(m_{0}\right) .
$$

Here $\mathbf{n}(\mathrm{m})$ is the unit vector of the gradient of material $\mathrm{m}$. Then we order the materials according to the value of $p(m)$. After the normal direction and order of materials are all determined, we move the interfaces of materials along the normal direction until each material matches its volume in the cell. We would like to point out that until recently we had used a similar formula to Eq.(5) in Roxane [34] for the two-dimensional case except for the square root.

Due to the variations of the normal direction, the procedure stated above is tedious in implementation in the three-dimensional case. Figure 5 shows a two-dimensional mesh with four materials and ten materials, and Fig.6 is for a three-dimensional case in which each color represents one material. In Figs.5 and 6, only mixed cells are displayed.

\section{Numerical Schemes}

Since resulting meshes through reconstruction of interfaces between materials are unstructured that include arbitrary polygons and polyhedrons, we will discuss numerical schemes for Eq.(1) on 


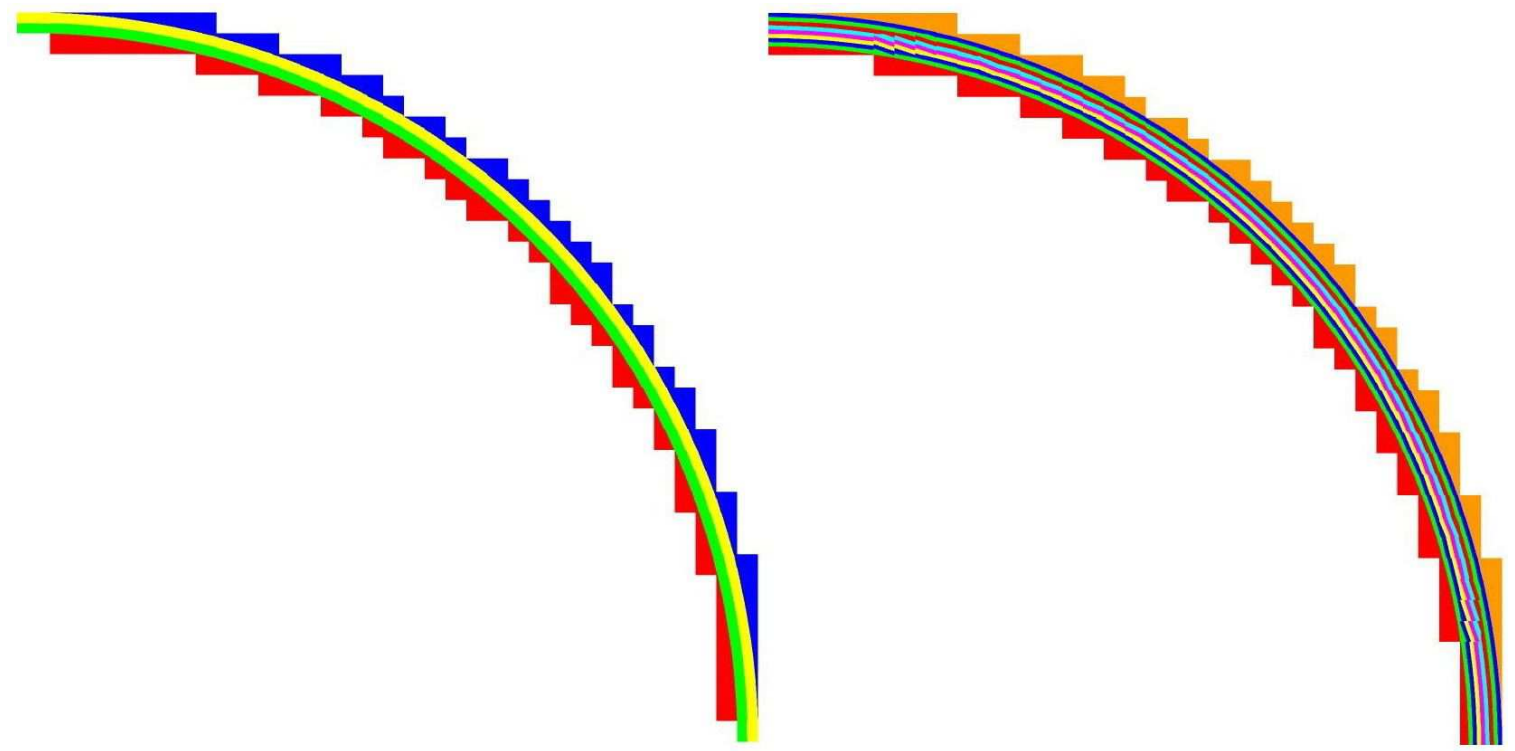

Figure 5: The reconstructed material interfaces of four materials (left image) and ten materials (right image) in two dimensions.

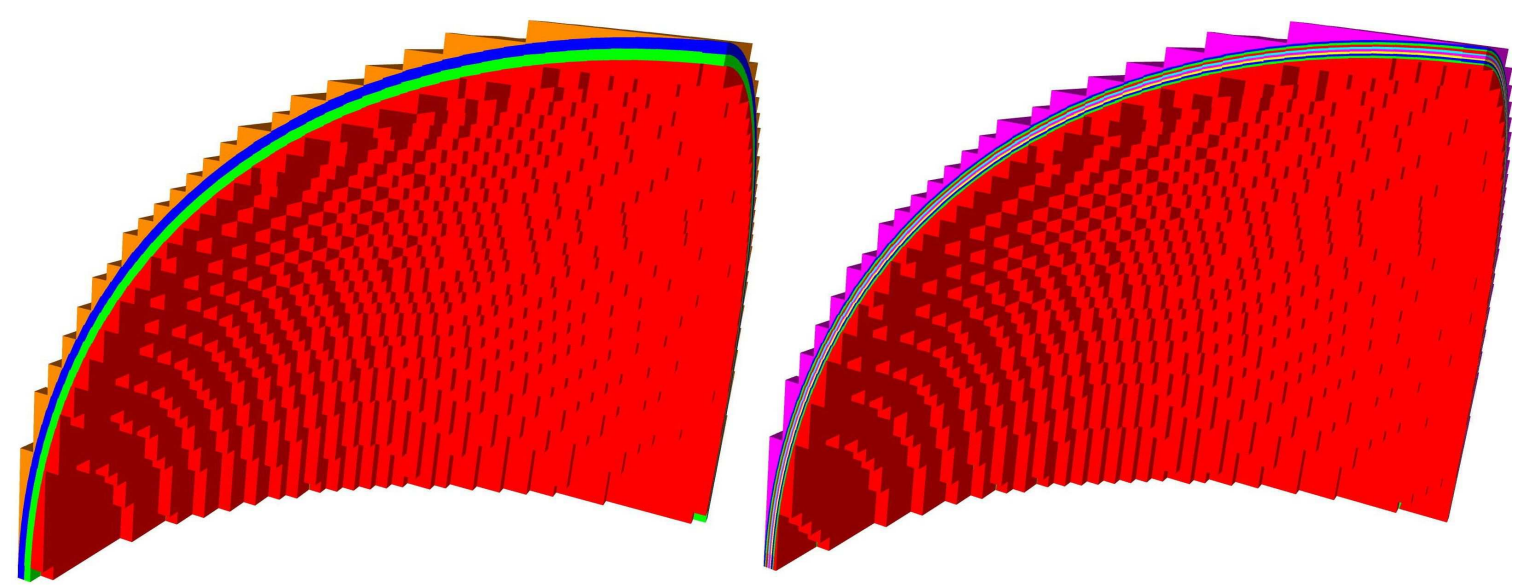

Figure 6: The reconstructed material interfaces of four materials (left image) and ten materials in three dimensions. 
a general polyhedral mesh in two and three dimensions. After we develop our numerical scheme on unstructured meshes, we will simplify apply the scheme to polyhedral meshes generated from reconstruction of material interface.

\subsection{Second-order Accuracy in Time}

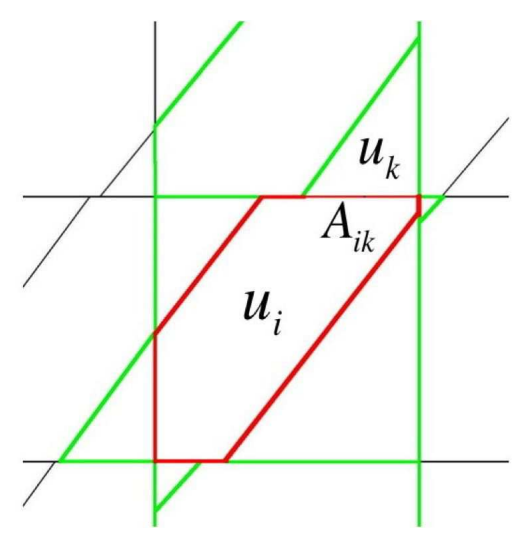

Figure 7: The cell $i$ (the red) and its neighboring cells (the green). $A_{i k}$ is the area between cell $i$ and one of its neighboring cells, cell $k$

In the Fig.7, we illustrate a cell of an unstructured mesh, the red cell, which thermally interacts with its neighboring cells marked with the green color through thermal flux across the interface between the red cell and each green cell. For example, the $i^{\text {th }}$ cell interacts with $k^{\text {th }}$ cell through the interface $A_{i k}$. The interface $A_{i k}$ is one whole face of the $k^{t h}$ cell and a part of a face in the $i^{\text {th }}$ cell. Considering the red cell as shown in Fig.7, we integrate Eq.(1) over the cell and one time step $0<t<\Delta t$ with $\triangle t$ the size of time step. After converting the volume integral into a surface integral over the enclosed surface of the cell for the term of flux, we get the following equation,

$$
u_{i}^{n}=u_{i}-\frac{\triangle t}{\triangle V_{i}} \sum_{k \in N_{i}} \bar{F}_{i k} A_{i k}+\bar{S}_{i} \triangle t .
$$

Here $\triangle V_{i}$ is the volume of the cell, $N_{i}$ is a set of cells, each of which neighbors the cell $i$ with a common non-vanishing interface as shown by the green cells in Fig.7. $A_{i k}$ is the area of the interface between cell $i$ and cell $k . \bar{S}_{i}$ is the source term defined as

$$
\bar{S}_{i} \equiv \frac{1}{\triangle t \triangle V_{i}} \int_{0}^{\triangle t} \int_{\triangle V_{i}} S(t, \mathbf{r}) d V d t
$$

The superscript $n$ in Eq.(6) stands for the new time $\Delta t . u_{i}^{n}$ is the cell averaged $u$ at $t=\Delta t$ and it is defined as

$$
u_{i}^{n} \equiv \frac{1}{\triangle V_{i}} \int_{\triangle V_{i}} u(\triangle t, \mathbf{r}) d V
$$

$u_{i}$ is similarly defined at $t=0 . \bar{F}_{i k}$ is the time-averaged flux across the common interface $A_{i k}$ and is defined as

$$
\bar{F}_{i k} \equiv \frac{1}{A_{i k} \triangle t} \int_{0}^{\triangle t} \int_{A_{i k}} \mathbf{F} \cdot d \mathbf{a} d t
$$


The summation in Eq.(6) is over the set $N_{i}$, the neighboring cells of cell $i$. We would like to point out that Eq.(6) is exact, and we have not made any approximation yet.

If we know how to calculate the time-averaged flux needed in Eq.(6), we then obtain $u$ at $t=\triangle t$ from the given initial condition and source function. Therefore, one of the major tasks in numerical methods is to approximately find the time-averaged flux.

If the time-averaged flux is replaced by its initial value, the approximation results in Euler forward method. If the flux is replaced by the value at the end of the time step, the scheme is called Euler backward scheme. Both Euler forward and backward schemes are first order accurate in time. One of the good features of Euler backward scheme is that numerical errors undergo quick damping for large time steps, and this feature is very useful for steady states, and it is important for a time-dependent problem if materials in the problem have very different properties. If the time-average flux in Eq.(6) is replaced by the averaged of fluxes at $t=0$ and $t=\Delta t$, the result is the Crank-Nicolson scheme, which is second order accurate in time. But the numerical errors in Crank-Nicolson scheme do not damp out for large time steps.

To introduce quick damping for numerical errors within the second order accuracy, we introduce an additional time level, as demonstrated in the scheme for hydrodynamics [5,7], for radiation hydrodynamics [16], and for heat conduction on structured mesh [12]. Within the second order accuracy, we approximately evaluate the time-averaged flux at $t=\triangle t / 2$. Thus Eq.(6) becomes

$$
u_{i}^{n}=u_{i}-\frac{\triangle t}{\triangle V_{i}} \sum_{k \in N_{i}} F_{i k}^{h} A_{i k}+S_{i}\left(T_{i}^{h}\right) \triangle t .
$$

Here the superscript $h$ stands for the evaluation at the half time step, $t=\Delta t / 2 . F_{i k}^{h}$ is the flux evaluated at the half time step and at the interface between cells $i$ and $k$, defined as

$$
F_{i k}^{h} \equiv \frac{1}{A_{i k}} \int_{A_{i k}} \mathbf{F}(\triangle t / 2, \mathbf{r}) \cdot d \mathbf{a}
$$

There are a few approaches that can be used to approximately calculate $F_{i k}^{h}$. For example, $F_{i k}^{h}$ could be approximately calculated through $\left(T_{i}+T_{i}^{h}\right) / 2$ or $\left(F_{i k}+F_{i k}^{n}\right) / 2$, here again the superscript $n$ stands for the evaluation at the new time $t=\Delta t$. But the resulting scheme through this will not work for large time steps, although it is second order accurate.

Our approach is to introduce a new set of unknowns at $t=\Delta t / 2, u_{i}^{h}$ or $T_{i}^{h}$, which are defined as

$$
u_{i}^{h} \equiv \frac{1}{\triangle V_{i}} \int_{\triangle V_{i}} u(\triangle t / 2, \mathbf{r}) d V .
$$

Since $u_{i}^{h}$ or $T_{i}^{h}$ is a new set of unknowns, like $u_{i}^{h}$ or $T_{i}^{n}$, we need an equation to determine $u_{i}^{h}$ or $T_{i}^{h}$. For this purpose, we integrate Eq.(1) on the volume $\triangle V_{i}$ and the first half time step $0 \leq t \leq \Delta t / 2$. After converting the volume integral of the flux into surface integral, we obtain the following,

$$
u_{i}^{h}=u_{i}-\frac{\triangle t}{2 \triangle V_{i}} \sum_{k \in N_{i}} \bar{F}_{i k}^{h} A_{i k}+\frac{1}{2} \bar{S}_{i}^{h} \triangle t .
$$

Here $\bar{F}_{i k}^{h}$ and $\bar{S}_{i}^{h}$ are defined as

$$
\bar{F}_{i k}^{h} \equiv \frac{2}{\triangle t A_{i k}} \int_{0}^{\triangle t / 2} \int_{A_{i k}} \mathbf{F} \cdot d \mathbf{a} d t .
$$




$$
\bar{S}_{i}^{h} \equiv \frac{2}{\triangle t \triangle V_{i}} \int_{0}^{\triangle t / 2} \int_{\triangle V_{i}} S(t, \mathbf{r}) d V d t .
$$

The time-averaged flux Eq.(13) may be approximately calculated through an interpolation in time. But, as stated before, an approximate calculation for the time-averaged flux must not, even partially, depend on the initial temperature if we want the property of the scheme for large time steps. Therefore, instead of interpolation through $F_{i k}$ (the initial flux) and $F_{i k}^{n}$, we use extrapolation through $F_{i k}^{h}$ and $F_{i k}^{n}$ to find $\bar{F}_{i k}^{h}$. Therefore the linear form of $F_{i k}(t)$ with time is uniquely determine by its values at $t=\triangle t / 2$ and $t=\triangle t, F_{i k}^{h}$ and $F_{i k}^{n}$. Thus, the time-averaged flux and sources defined in Eq.(13) is approximately obtained through the following approximation:

$$
\begin{gathered}
\bar{F}_{i k}^{h} \approx \frac{3}{2} F_{i k}^{n}-\frac{1}{2} F_{i k}^{h}, \\
\bar{S}_{i}^{h} \approx \frac{3}{2} S_{i}^{n}-\frac{1}{2} S_{i}^{h} .
\end{gathered}
$$

Here $F_{i k}^{n}$ is the flux calculated from temperature $T_{i}^{n}$ at $t=\triangle t, F_{i k}^{h}$ is the flux calculated from temperature $T_{i}^{h}$ at $t=\triangle t / 2, S_{i}^{n}$ and $S_{i}^{h}$ are source term $S(T)$ evaluated at $T_{i}^{n}$ and $T_{i}^{h}$, and $\bar{S}_{i}^{n}$ is

a function of $T_{i}^{h}$, and $\bar{S}_{i}^{h}$ is a function of both $T_{i}^{n}$ and $T_{i}^{h}$. Except for the calculation of flux from temperature, we have developed our difference equations as shown in Eqs. $(9,12)$ with Eqs. $(14,15)$ for the diffusion equation (1). We have to mention that this kind of time-stepping has also been developed in [28].

\subsection{Effective Diffusion Coefficients across Material Interface}

Now we move to the calculation of the flux from temperature needed in Eqs. $(9,12)$. As we stated before, there are possibly discontinuities in diffusion coefficient between cells, and thus spatial derivatives is discontinuous across the interfaces although temperature is continuous. Therefore, we can not use Taylor expansion across interface to approximately evaluate the flux if there is a strong discontinuity across an interface.

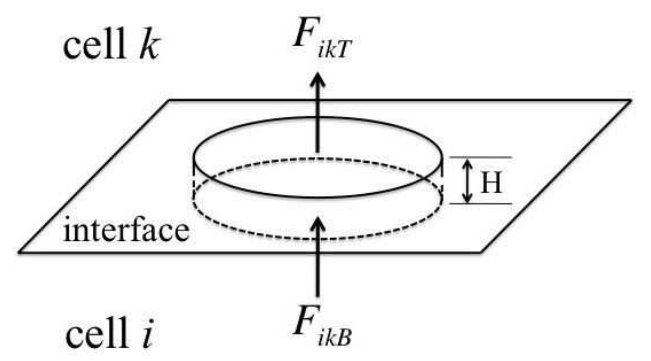

Figure 8: Illustration of the relationship of energy flux between two sides of interface of cells $\mathrm{i}$ and $\mathrm{k}$. The short cylinder is crossing the interface between cells $\mathrm{i}$ and $\mathrm{k}$. $\mathrm{H}$ is the height of the cylinder which is taking the limit to zero.

Our calculation for the flux is based on the conservation law of energy. We integrate Eq.(1) over a small disk across the interface between cells i and k, as shown in Fig.8, and we let the height of the disk goes vanishing as the limit. We have 


$$
F_{i k T}=F_{i k B}+\text { surface density of source. }
$$

Here $F_{i k T}$ is the flux evaluated at cell $k$ side, and $F_{i k B}$ is the one evaluated at the side of cell $i$. Since we do not have any surface source, we have $F_{i k T}=F_{i k B}$. Also, $F_{i k T}$ and $F_{i k B}$ each is $F_{i k}$, the flux across the interface. To calculate $F_{i k}$, we use $T_{i k *}$ to represent the temperature at the center of the interface between cells $i$ and $k$, as shown in Fig.9, $\mathbf{n}_{i k}$ is the normal direction of the interface between cells $i$ and $k$, from cell $i$ to cell $k, \mathbf{l}_{i *}$ is the distance vector from the center of cell $i$ to the center of the interface, $l_{i *}$ is its magnitude, $\mathbf{n}_{i *}$ is the unit vector along $\mathbf{l}_{i *}$, ie, $\mathbf{n}_{i *} \equiv \mathbf{l}_{i *} / l_{i *}$. We approximately evaluate flux from the cell center of cell $i$ to the center of interface as

$$
\mathbf{F}_{i k B}=-\frac{\kappa\left(T_{i}\right)}{l_{i *}}\left(T_{i k *}-T_{i}\right) \mathbf{n}_{i *}
$$

Here $\kappa$ is defined as

$$
\kappa(T)=\kappa_{0}(T)+4 T^{3} \sigma(T) .
$$

Therefore, the flux across the interface along the normal direction is

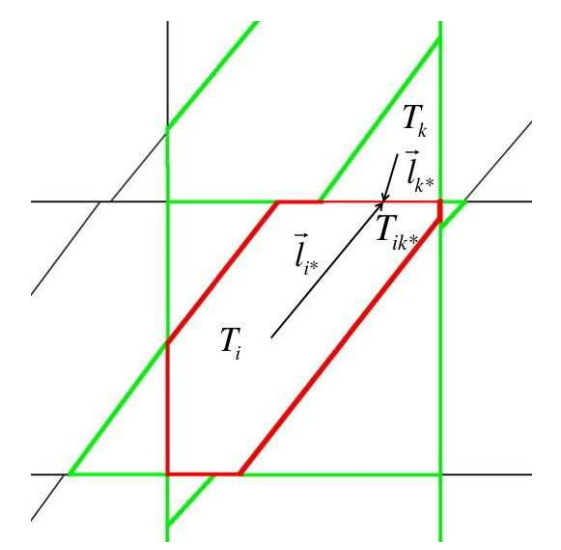

Figure 9: Illustration of the procedure to calculate energy flux across the interface between cells $i$ and $k . l_{i *}$ or $l_{k *}$ is the vector from the center of cell $i$ or $k$ to the center of the interface. $T_{i}$ and $T_{k}$ are the temperatures at the centers of cells $i$ and $k$ respectively. $T_{i k *}$ is the temperature at the center of the interface.

$$
F_{i k B} \approx-\frac{\kappa_{i}}{l_{i *}}\left(T_{i k *}-T_{i}\right) \alpha_{i}
$$

Here $\alpha_{i}$ is the component of $\mathbf{n}_{i *}$ along the normal direction, $\alpha_{i} \equiv \mathbf{n}_{i *} \cdot \mathbf{n}_{i k}>0$. Similarly, we calculate the flux $F_{i k}$ from the side of cell $k$,

$$
F_{i k T} \approx-\frac{\kappa_{k}}{l_{k *}}\left(T_{k}-T_{i k *}\right) \alpha_{k}
$$

Here $\mathbf{l}_{k *}$ is the distance vector from the center of cell $k$ to the center of the interface, $l_{k *}$ is its magnitude, $\mathbf{n}_{k *}$ is the unit vector along $\mathbf{l}_{k *}, \mathbf{n}_{k *} \equiv \mathbf{l}_{k *} / l_{k *}$, and $\alpha_{k}$ is the component of $-\mathbf{n}_{k *}$ along the normal direction, $\alpha_{k} \equiv-\mathbf{n}_{k} \cdot \mathbf{n}_{i k}>0$. Two equations, Eqs.(16,17) must be equal. Thus from Eqs. $(16,17)$ we can write $T_{i k *}$ as

$$
T_{i k *}=\frac{l_{k *} \alpha_{i} \kappa_{i} T_{i}+l_{i *} \alpha_{k} \kappa_{k} T_{k}}{l_{k *} \alpha_{i} \kappa_{i}+l_{i *} \alpha_{k} \kappa_{k}}
$$


Here $\kappa_{i}$ and $\kappa_{k}$ are $\kappa$ evaluated at cells $i$ and $k$ respectively. Substituting this $T_{i k *}$ into Eq.(16) or Eq.(17) we get the flux across the interface

$$
F_{i k}=-\tilde{\kappa}_{i k}\left(T_{k}-T_{i}\right),
$$

with definition

$$
\tilde{\kappa}_{i k} \equiv \frac{\kappa_{i} \kappa_{k}}{l_{k *} \alpha_{i} \kappa_{i}+l_{i *} \alpha_{k} \kappa_{k}} \alpha_{i} \alpha_{k} .
$$

We would like to point out that the diffusion coefficients $\kappa_{i}$ and $\kappa_{k}$ are values defined on cells. Typically, they are calculated from analytical formulae or obtained from lookup tables for given values of material density and temperature on the cells.

\subsection{Difference Equations on General Polyhedral Meshes}

To get difference equations for the diffusion equation, we substitute the flux Eq.(19) into Eqs.(9,12,14), and get

$$
\begin{aligned}
& u_{i}^{n}+\frac{\triangle t}{\triangle V_{i}}\left(\sum_{k \in N_{i}} \tilde{\kappa}_{i k}^{h} A_{i k}\right) T_{i}^{h}=u_{i}+\frac{\triangle t}{\triangle V_{i}} \sum_{k \in N_{i}}\left(\tilde{\kappa}_{i k}^{h} A_{i k} T_{k}^{h}\right)+S_{i}^{h} \triangle t, \\
& -\frac{\triangle t}{4 \triangle V_{i}}\left(\sum_{k \in N_{i}} \tilde{\kappa}_{i k}^{n} A_{i k}\right) T_{i}^{n}+u_{i}^{h}+\frac{3 \triangle t}{4 \triangle V_{i}}\left(\sum_{k \in N_{i}} \tilde{\kappa}_{i k}^{h} A_{i k}\right) T_{i}^{h} \\
& =u_{i}+\frac{3 \triangle t}{4 \triangle V_{i}} \sum_{k \in N_{i}}\left(\tilde{\kappa}_{i k}^{h} A_{i k} T_{k}^{h}\right)-\frac{\triangle t}{4 \triangle V_{i}} \sum_{k \in N_{i}}\left(\tilde{\kappa}_{i k}^{n} A_{i k} T_{k}^{n}\right)+\frac{1}{2} \bar{S}_{i}^{h} \triangle t .
\end{aligned}
$$

Here the superscripts $n$ and $h$ of $\tilde{\kappa}_{i k}^{n}$ and $\tilde{\kappa}_{i k}^{h}$ stand for $\tilde{\kappa}_{i k}\left(T_{i}^{n}, T_{k}^{n}\right)$ and $\tilde{\kappa}_{i k}\left(T_{i}^{h}, T_{k}^{h}\right)$. Equations $(21,22)$ are our difference scheme, which applies to general polyhedral meshes in one-, two-, and three dimensions, and is second order accurate in space and time.

The set of Eqs. $(21,22)$ is nonlinear due to the nonlinear dependence of $u$ on $T$ and the dependence of $\kappa$ on $T, \kappa=\kappa(T)$. We linearize this set of equations before we apply iterative solvers, for example, Hypre, a library developed by Lawrence Livermore National Laboratory. Thus, we get a set of linear equations

$$
\begin{gathered}
\hat{u}_{i} T_{i}^{n}+\left[-\hat{S}_{i}+\frac{\triangle t}{\triangle V_{i}}\left(\sum_{k \in N_{i}} \tilde{\kappa}_{i k} A_{i k}\right)\right] T_{i}^{h}=\hat{u}_{i} T_{i}+\frac{\triangle t}{\triangle V_{i}} \sum_{k \in N_{i}}\left(\tilde{\kappa}_{i k} A_{i k} T_{k}^{h}\right)+S_{i}^{0} \triangle t \\
-\left[\hat{s}_{i}^{n}+\frac{\triangle t}{4 \triangle V_{i}}\left(\sum_{k \in N_{i}} \tilde{\kappa}_{i k} A_{i k}\right)\right] T_{i}^{n}+\left[-\hat{s}_{i}^{h}+\hat{u}_{i}+\frac{3 \triangle t}{4 \triangle V_{i}}\left(\sum_{k \in N_{i}} \tilde{\kappa}_{i k} A_{i k}\right)\right] T_{i}^{h} \\
=\hat{u}_{i} T_{i}+\frac{3 \triangle t}{4 \triangle V_{i}} \sum_{k \in N_{i}}\left(\tilde{\kappa}_{i k} A_{i k} T_{k}^{h}\right)-\frac{\triangle t}{4 \triangle V_{i}} \sum_{k \in N_{i}}\left(\kappa_{i k} A_{i k} T_{k}^{n}\right)+\frac{1}{2} \bar{s}_{i}^{0} \triangle t .
\end{gathered}
$$

Here $\kappa_{i k}$ is evaluated at the initial temperature $T_{i}$ and $T_{k}$ as defined in Eq.(20). $\hat{u}_{i}$ is defined as

$$
\hat{u}_{i} \equiv C_{v i}+4 a T_{i}^{3}
$$


$S_{i}^{0}, \hat{S}_{i}, s_{i}^{0}, \hat{s}_{i}^{h}$, and $\hat{s}_{i}^{n}$ are the coefficients from the linear expansions of source terms $S_{i}\left(T_{i}^{h}\right)$ and $\bar{S}_{i}^{h}\left(T_{i}^{n}, T_{i}^{h}\right)$,

$$
\begin{gathered}
S_{i}^{h} \approx S_{i}^{0}+\hat{S}_{i} T_{i}^{h}, \\
\bar{S}_{i}^{h} \approx s_{i}^{0}+\hat{s}_{i}^{h} T_{i}^{h}+\hat{s}_{i}^{n} T_{i}^{n} .
\end{gathered}
$$

The set of equations, Eqs. $(23,24)$, forms a linear system with $N+N$ unknowns, $T_{i}^{n}$ and $T_{i}^{h}, i=$ $1,2, \ldots, N$. Here $N$ is the number of cells. Eqs. $(23,24)$ are iteratively solved. One straightforward iterative approach is as follows. For the given set of $T_{i}$, we initial guess $T_{i}^{n}$ and $T_{i}^{h}$ as $T_{i}, i=1$, 2, ... $N$. Then for each $i$, using most recent values of $T_{i}^{n}$ and $T_{i}^{h}$, we evaluate the right sides of Eqs. $(23,24)$. After that we solve Eqs. $(23,24)$ for $T_{i}^{n}$ and $T_{i}^{h}$, to get improved $T_{i}^{n}$ and $T_{i}^{h}$. After doing this for all $i, i=1,2, \ldots, N$, we will have finished one iteration. This primitive iterative approach converges, although it is slow. We can also use existing linear solvers to solve Eqs.(23,24).

In our code, we use an existing algebraic multigrid (AMG) solver by default. The primitive iterative approach described above is in our code as a second option. Actually, the primitive solver has a few advantages compared with AMG solvers. First, we dont have to form any matrix in the primitive solver. Secondly, the primitive solver involves only point-to-point communications between neighboring computer processors. AMG solvers involve much more broad communications in coarsened grids and involve several global communications in the coarsest level. Actually, the primitive solver is not much slower than AMG if problems are not too large, for example, the problems on 64 processors. Typically the primitive solver costs 10\% to 100\% more compared with the AMG solver we use. For larger problems, we anticipate that AMG solvers will be much faster. It is our recommendation for users to use some multigrid solvers for the linearized Eqs. $(23,24)$.

\subsection{Numerical Scheme on Structured Meshes with AMR}

The formula and scheme developed in Section 4.1 are general for general polyhedral meshes. In this subsection, we will reduce the scheme for a special case, structured meshes with AMR, in which each cell has a rectangular shape.
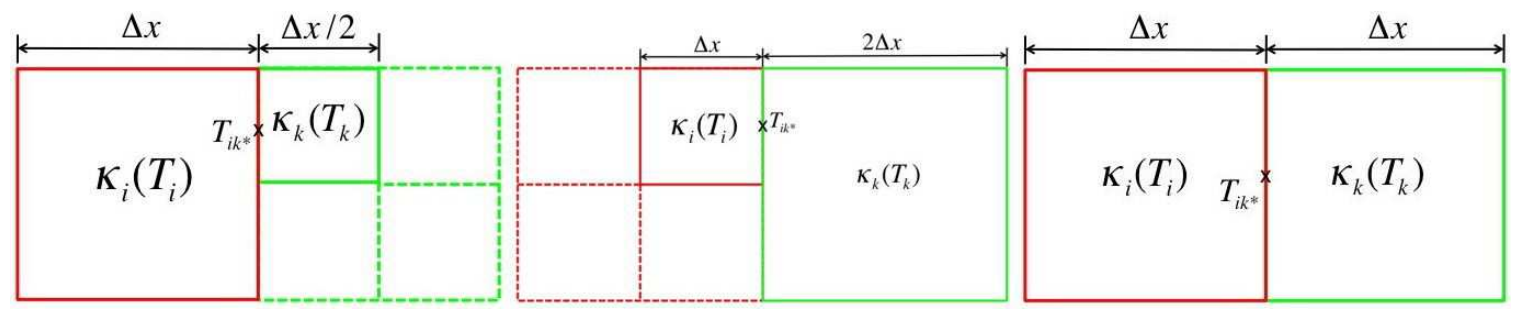

Figure 10: Three cases of a cell $i$ in structured mesh AMR: neighboring with a finer cell $k$ (left), with a corser cell (middle), and with a cell at the same refinement level (right).

For the cells $i$ and $k$ as shown in the left image of Fig.10, the temperature at a cell interface, Eq.(18), becomes

$$
\begin{aligned}
T_{i k *} & =\frac{\kappa_{i} T_{i}+3 \kappa_{k} T_{k}}{\kappa_{i}+3 \kappa_{k}} \text { for } 3 D . \\
T_{i k *} & =\frac{2 \kappa_{i} T_{i}+5 \kappa_{k} T_{k}}{2 \kappa_{i}+5 \kappa_{k}} \text { for } 2 D .
\end{aligned}
$$


For the cells $i$ and $k$ as shown in the right image of Fig.10,

$$
T_{i k *}=\frac{\kappa_{i} T_{i}+\kappa_{k} T_{k}}{\kappa_{i}+\kappa_{k}}
$$

The effective diffusion coefficient, measured by the size of cell $i$, Eq.(20) becomes

$$
\tilde{\kappa}_{i k}=\frac{\kappa_{i k}}{\triangle x} .
$$

Here $\kappa_{i k}$ is different for different kinds of neighboring cell $k$ and $\triangle x$ is the width of the cell $i$. For the neighbor cell $k$ as shown in the left image of Fig.10, it is

$$
\begin{aligned}
\kappa_{i k}^{(c)} & =\frac{4 \kappa_{i} \kappa_{k}}{\kappa_{i}+3 \kappa_{k}} \text { for } 3 D . \\
\kappa_{i k}^{(c)} & =\frac{8 \kappa_{i} \kappa_{k}}{2 \kappa_{i}+5 \kappa_{k}} \text { for } 2 D .
\end{aligned}
$$

For the cells $i$ and $k$ shown in the middle image of Fig.10,

$$
\begin{aligned}
\kappa_{i k}^{(p)} & =\frac{2 \kappa_{i} \kappa_{k}}{3 \kappa_{i}+\kappa_{k}} \text { for } 3 D . \\
\kappa_{i k}^{(p)} & =\frac{4 \kappa_{i} \kappa_{k}}{5 \kappa_{i}+2 \kappa_{k}} \text { for } 2 D .
\end{aligned}
$$

For the cells $i$ and $k$ shown in the image at the right of Fig.10,

$$
\kappa_{i k}^{(b)}=\frac{2 \kappa_{i} \kappa_{k}}{\kappa_{i}+\kappa_{k}} .
$$

This formula is the same as previous investigations, for example, [11]. The difference equations, Eqs. $(23,24)$, become

$$
\begin{aligned}
& \hat{u}_{i} T_{i}^{n}+\frac{\triangle t}{\triangle x_{i}}\left[\sum_{k \in B_{i}} \kappa_{i k}^{(b)}+\sum_{k \in P_{i}} \kappa_{i k}^{(p)}+\sum_{k \in C_{i}} \kappa_{i k}^{(c)}\right] T_{i}^{h} \\
= & \hat{u}_{i} T_{i}+\frac{\triangle t}{\triangle x_{i}}\left[\sum_{k \in B_{i}} \kappa_{i k}^{(b)} T_{k}^{h}+\sum_{k \in P_{i}} \kappa_{i k}^{(p)} T_{k}^{h}+\sum_{k \in C_{i}} \kappa_{i k}^{(c)} T_{k}^{h}\right]+S_{i}^{h} \triangle t \\
& -\frac{\triangle t}{4 \triangle x_{i}}\left[\sum_{k \in B_{i}} \kappa_{i k}^{(b)}+\sum_{k \in P_{i}} \kappa_{i k}^{(p)}+\sum_{k \in C_{i}} \kappa_{i k}^{(c)}\right] T_{i}^{n} \\
& +\left[\hat{u}_{i}+\frac{3 \triangle t}{4 \triangle x_{i}}\left(\sum_{k \in B_{i}} \kappa_{i k}^{(b)}+\sum_{k \in P_{i}} \kappa_{i k}^{(p)}+\sum_{k \in C_{i}} \kappa_{i k}^{(c)}\right)\right] T_{i}^{h} \\
& =\hat{u}_{i} T_{i}+\frac{3 \triangle t}{4 \triangle x_{i}}\left[\sum_{k \in B_{i}} \kappa_{i k}^{(b)} T_{k}^{h}+\sum_{k \in P_{i}} \kappa_{i k}^{(p)} T_{k}^{h}+\sum_{k \in C_{i}} \kappa_{i k}^{(c)} T_{k}^{h}\right] \\
& -\frac{\triangle t}{4 \triangle x_{i}}\left[\sum_{k \in B_{i}} \kappa_{i k}^{(b)} T_{k}^{n}+\sum_{k \in P_{i}} \kappa_{i k}^{(p)} T_{k}^{n}+\sum_{k \in C_{i}} \kappa_{i k}^{(c)} T_{k}^{n}\right]+\frac{1}{2} \bar{S}_{i}^{h} \triangle t .
\end{aligned}
$$

Here $B_{i}$ or $C_{i}$ or $P_{i}$ stands for those neighboring cells of cell $i$ that has the same refinement level as cell $i$ (at the same refinement level), or are one level coarser (at the child level)) or finer than the 
cell $i$ (at the parent level), $\triangle x_{i}$ is the width of the cell $i$. In Eqs.(33,34) we have also assumed that each cell is a cube, ie, all sides of each cell are equal in length. If a cell is not cube, one is easy to get the equivalent equations from Eqs. $(23,24)$.

If the cell $i$ does not have finer or coarser neighboring cells, the set $P_{i}$ or $C_{i}$ is empty. If the cell $i$ does not have neighboring cells that is at the same refinement level, $B_{i}$ is empty. The formula will be simpler for a uniform structured mesh. For this case the difference equations, Equations $(33,34)$, turn to

$$
\begin{aligned}
& \hat{u}_{i} T_{i}^{n}+\frac{\triangle t}{\triangle x_{i}}\left(\sum_{k \in B_{i}} \kappa_{i k}^{(b)}\right) T_{i}^{h}=\hat{u}_{i} T_{i}+\frac{\triangle t}{\triangle x_{i}} \sum_{k \in B_{i}}\left(\kappa_{i k}^{(b)} T_{k}^{h}\right)+S_{i}^{h} \triangle t, \\
& -\frac{\triangle t}{4 \triangle x_{i}}\left(\sum_{k \in B_{i}} \kappa_{i k}^{(b)}\right) T_{i}^{n}+\left[\hat{u}_{i}+\frac{3 \triangle t}{4 \triangle x_{i}} \sum_{k \in B_{i}} \kappa_{i k}^{(b)}\right] T_{i}^{h} \\
& =\hat{u}_{i} T_{i}+\frac{3 \triangle t}{4 \triangle x_{i}} \sum_{k \in B_{i}}\left(\kappa_{i k}^{(b)} T_{k}^{h}\right)-\frac{\triangle t}{4 \triangle x_{i}} \sum_{k \in B_{i}}\left(\kappa_{i k}^{(b)} T_{k}^{n}\right)+\frac{1}{2} \bar{S}_{i}^{h} \triangle t .
\end{aligned}
$$

Here $\kappa_{i k}^{(b)}$ is given in Eq.(32) and $N_{i}$ is the set of cells that are neighboring with cell $i$.

\subsection{Steady States}

As stated before, we are interested in time-dependent problems. But, since materials involved in a problem may have very different diffusion coefficients so that a given time step may be so large for some material that formulations for steady states are more appropriate for the material. Our numerical scheme is designed to have the property that the steady states can be reached when a time step goes to infinite.

When a time step goes to infinite, since $T_{i}^{h}$ is the same $T_{i}^{n}$ the nonlinear difference equations, Eqs. $(21,22)$, turn to

$$
\left(\sum_{k \in N_{i}} \tilde{\kappa}_{i k}^{n} A_{i k}\right) T_{i}^{n}=\sum_{k \in N_{i}}\left(\tilde{\kappa}_{i k}^{n} A_{i k} T_{k}^{n}\right)+S_{i}\left(T_{i}^{n}\right) \triangle V_{i}
$$

This set of nonlinear equations is difficult or expensive to solve, but for the linear case it is much easier to solve

$$
\left(\sum_{k \in N_{i}} \kappa_{i k} A_{i k}\right) T_{i}^{n}=\sum_{k \in N_{i}}\left(\kappa_{i k} A_{i k} T_{k}^{n}\right)+S_{i}\left(T_{i}^{n}\right) \triangle V_{i}
$$

For structured meshes with AMR, the linear difference equations, Eqs.(33,34), become

$$
\left[\sum_{k \in B_{i}} \kappa_{i k}^{(b)}+\sum_{k \in P_{i}} \kappa_{i k}^{(p)}+\sum_{k \in C_{i}} \kappa_{i k}^{(c)}\right] T_{i}^{n}=\left[\sum_{k \in B_{i}} \kappa_{i k}^{(b)} T_{k}^{n}+\sum_{k \in P_{i}} \kappa_{i k}^{(p)} T_{k}^{n}+\sum_{k \in C_{i}} \kappa_{i k}^{(c)} T_{k}^{n}\right]+S_{i}\left(T_{i}^{n}\right) \triangle V_{i} .
$$

For uniform meshes, the linearized equation, Eqs.(35,36), turn to

$$
\left(\sum_{k \in B_{i}} \kappa_{i k}^{(b)}\right) T_{i}^{n}=\sum_{k \in B_{i}} \kappa_{i k}^{(b)} T_{k}^{n}+S_{i}\left(T_{i}^{n}\right) \triangle V_{i}
$$




\section{$5 \quad$ Numerical Examples}

In this section we will provide numerical examples to show the correctness and the features of the schemes for single and multi-materials. All the solutions are obtained through solving the linearized equations, Eqs.(23,24), or Eqs. $(35,36)$. The examples of steady states after only one time step is for linear problems. All the examples for nonlinear problems are time-dependent.

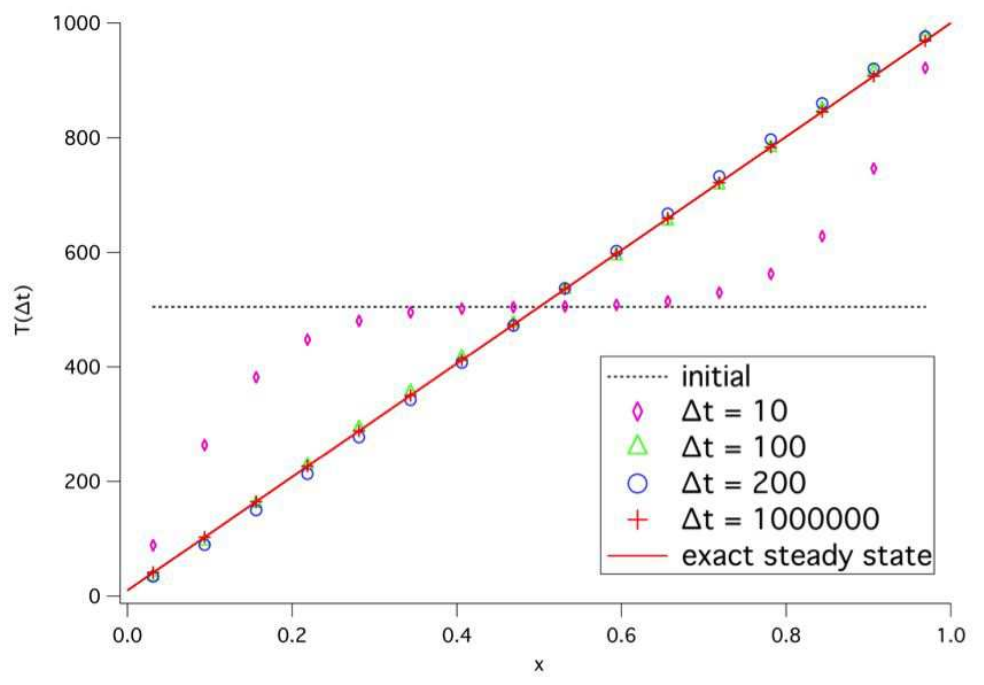

Figure 11: Four numerical solutions after one time step with four different sizes of time step. The dashed line is the initial condition, and the solid line is the exact steady state. Sixteen grid cells are used in these one-dimensional simulations.

The first example is to show how the solutions of the scheme change with different sizes of time step. Figure 11 shows four solutions of a linear diffusion problem, Eq.(1) with $\sigma=0$ and $C_{v}=1$ after one time step. The solutions are obtained from one-dimensional simulations with sixteen grid cells and fixed temperatures at $x=0$ and $x=1.0$. The dashed line is the initial distribution of temperature. As expected, the numerical solution after a very large time step is the correct steady state.

The second problem is to show the correctness when two materials are involved. Figure 12 shows a solution of a one-dimensional linear diffusion problem after a very large time step of a simulation with 32 grid cells, fixed temperatures at boundaries, and two materials, one with unity diffusion coefficient for $x<0.5$ and the other with diffusion coefficient 1000 for $x>0.5$. The markers with red crosses are the solution after one time step $\triangle t=10^{20}$ with effective diffusion coefficient, Eq.(32). The green line is the exact solution of steady state. For comparison, we also plotted the solution obtained from the simple algebraic average of diffusion coefficient. It should be pointed out that $\partial T / \partial x$ is discontinuous at $x=0.5$, but the flux is continuous. We have used this feature in the design of our numerical scheme. It is this feature that makes the scheme accurate for problems involving multi-materials.

The third example is to demonstrate the accuracy of the scheme. We are looking into a linear problem of Eq.(1) with $\kappa=1, \sigma=0$, and $C_{v}=1$. The initial and boundary conditions of the problem are $T(0, x)=1+\sin (2 \pi x), T(t, 0)=1$, and $T(t, 1)=1$. The exact solution of the problem 


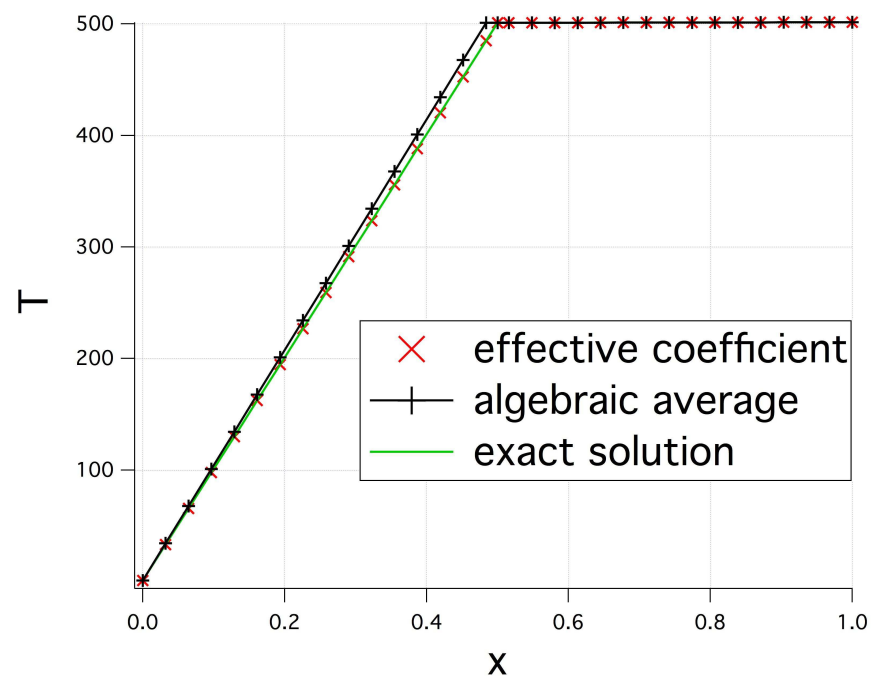

Figure 12: The solution (red crossings) after one large time step, $\triangle t=10^{20}$, for one-dimensional problem with two materials. Thirty two grid cells are used. The value at the material interface is calculated through Eq.(27). The green line is the exact steady state. The black line with plus markers is the solution obtained through a simple algebraic average for diffusion coefficient.

is

$$
T(t, x)=1+e^{-(2 \pi)^{2} \kappa t} \sin (2 \pi x) .
$$

Exact cell-average value $T_{i}(t)$ at time $\mathrm{t}$, as defined in Eq.(7), is

$$
T_{i}^{\text {exact }}(t)=1+\frac{\sin \left(\pi \triangle x_{i}\right)}{\pi \triangle x_{i}} \sin \left[\pi\left(x_{i}+x_{i+1}\right)\right] e^{-(2 \pi)^{2} \kappa t} .
$$

Here $\triangle x_{i}\left(\equiv x_{i+1}-x_{i}\right)$ is the width of a cell. To match the boundary condition exactly, we choose the center of the first cell $\left(x_{0}+x_{1}\right) / 2=0$, and the center of the last cell $\left(x_{N-1}+x_{N}\right) / 2=1$ with $N$ is the number of cells used. To measure the correctness of the numerical method, we define the error of numerical solution against the exact solution,

$$
\text { error }=\sum_{i=0}^{n}\left|T_{i}(t)-T_{i}^{\text {exact }}\right| \triangle x_{i} .
$$

Figure 13 shows the error at $t=0.1$ from different simulations with different space resolutions for fixed time step $\Delta t=10^{-8}$. From these data, we can see the second order accuracy of scheme for this problem under these space resolutions. To show the order of accuracy in time, we carried out another set of simulations with different sizes of time step but a fixed space resolution $N=4097$. Figure 14 shows the error of numerical solutions with different time resolutions. The figure shows that the accuracy in time is close to the second order. We have to point out the sudden change of the slope in this figure, although we don't know the reason.

The remaining set of examples is for time-dependent diffusion equations on polyhedral meshes resulting from reconstruction of material interfaces. In all these problems, reflection boundary condition is used. The first one of this set is a two-dimensional simulation on the domain $(0,9.6) \times$ $(0,9.6)$ with two materials separated at radius 5.5. The mesh has four levels of refinement at the 


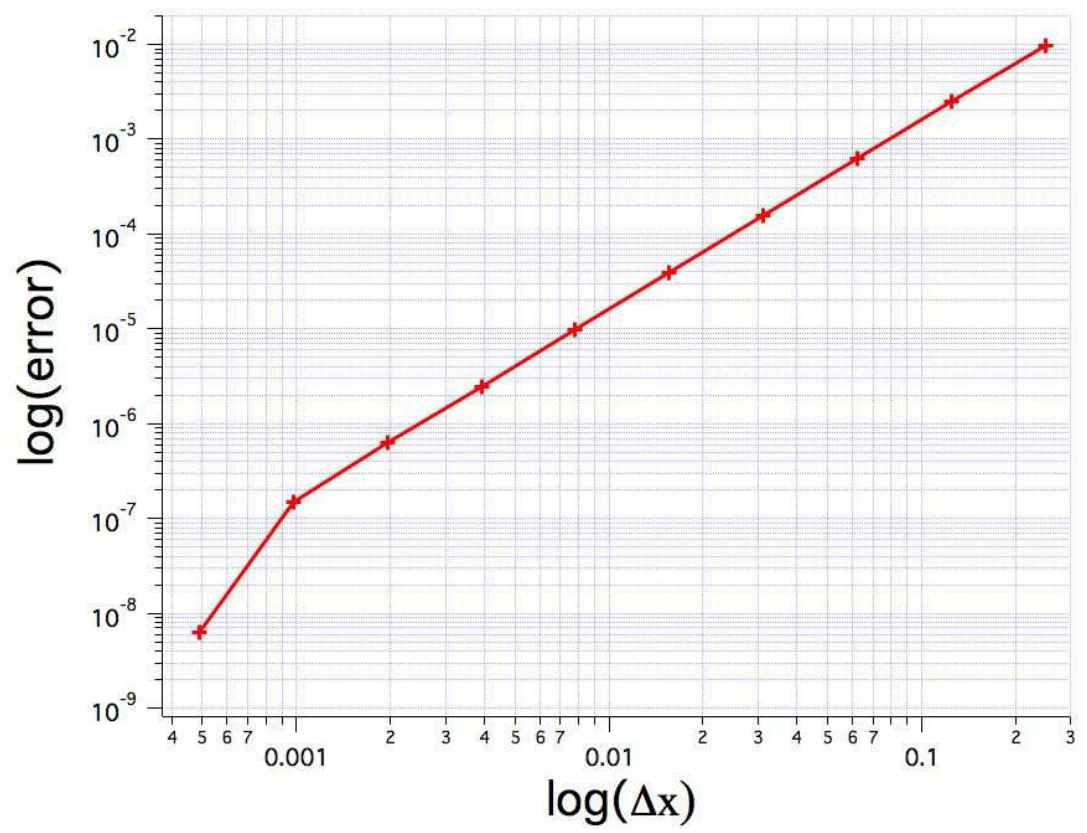

Figure 13: The error of numerical solutions at $\mathrm{t}=0.1$ vs different $\triangle x . \Delta t=10^{-8}$ in these simulations

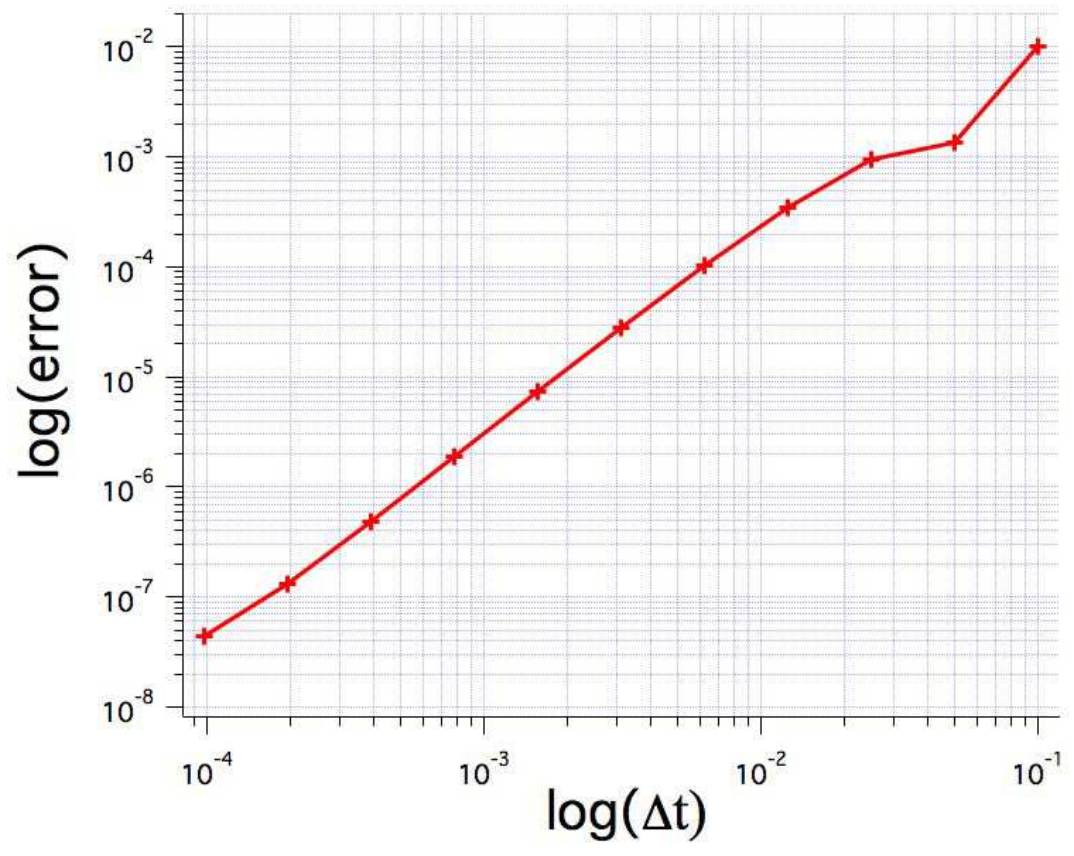

Figure 14: The error of numerical solutions at $t=0.1$ vs different $\triangle t$. The number of cells is 4097 . 

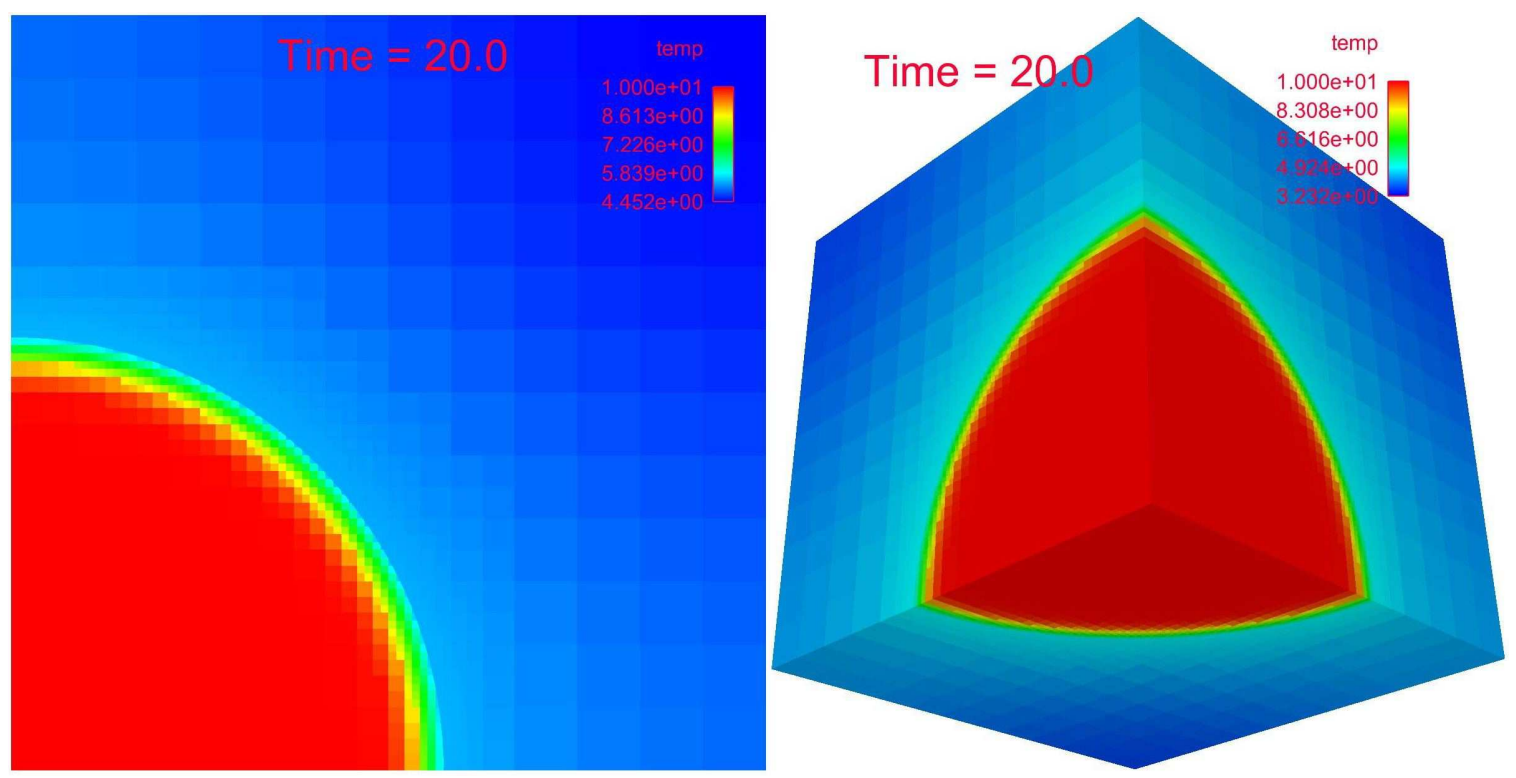

Figure 15: Numerical solutions at $t=20$ from two (the left image) and three (the right imgae) dimensional simulations.

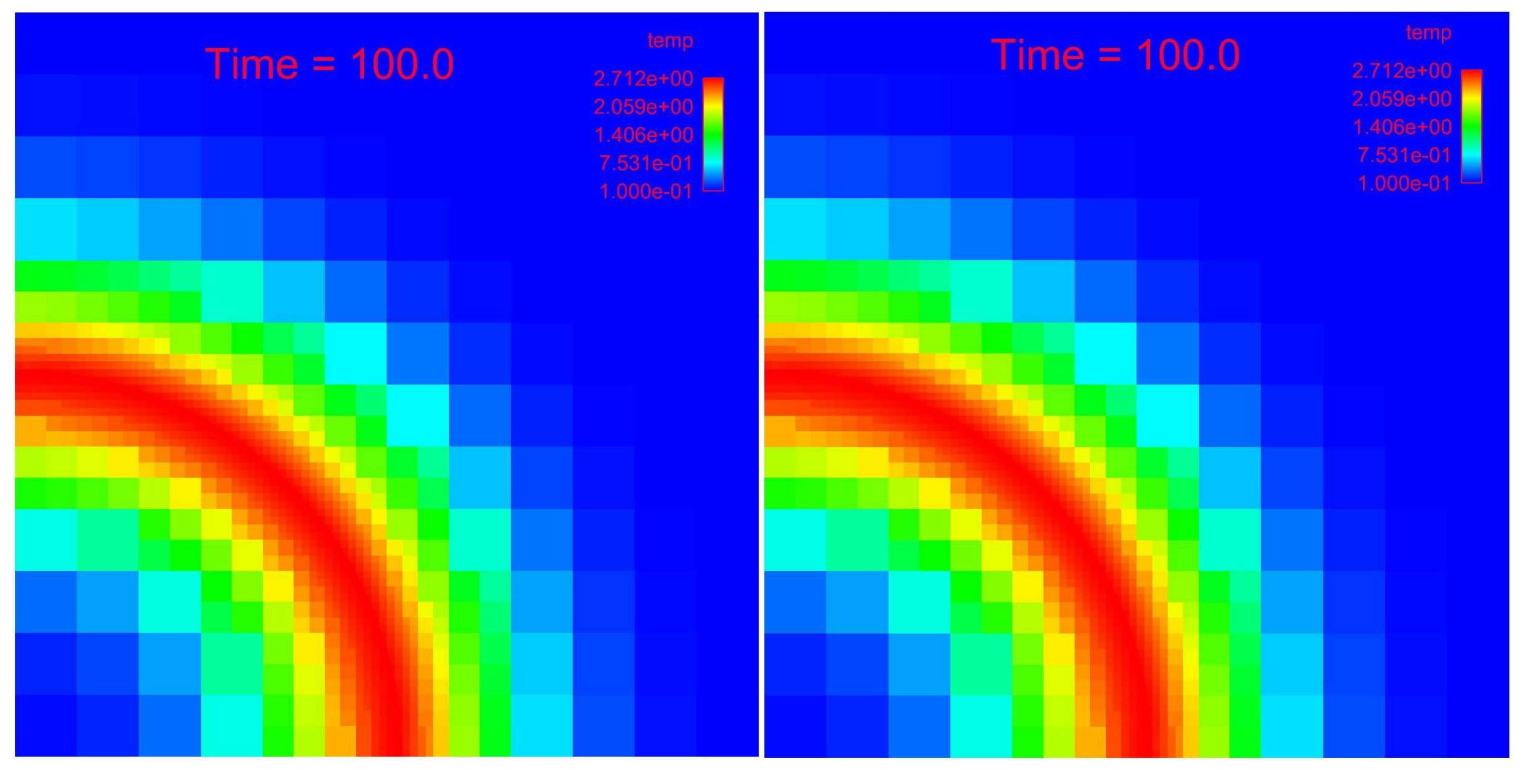

Figure 16: Numerical solutions at $t=100.0$ from a two dimensional simulation with fixed time step $\triangle t=0.001$ (the left image) and $\triangle t=1.0$ (the right image). 
material interface. The heat capacity, material diffusion coefficient, radiation diffusion coefficient, and initial temperature are 100,1000,0.1,10.0 in the inner region, and 1,10,100,0.01 in the outer region. The fixed time step 0.01 is used in this simulation. The left image in Figure 15 shows the temperature at $t=20$. The image at the right in Fig. 15 shows the solution of the problem extended to three dimensions.

Another example is for a two-dimensional problem with three materials separated at radius $r=4.9$ and $r=5.0$, in which the middle material has much large diffusion coefficient compared with the other two. In this example, $\sigma=0$ and $a=0$. The heat capacity $C_{v}$, material diffusion coefficient $\kappa_{0}$, and initial temperature are $(1,0.01,0.1),(10,100,10)$, and $(1,0.01,0.1)$ in the inner, middle, and outer materials. We run two simulations with different fixed time steps $\triangle t=0.001$ and $\triangle t=1.0$ respectively. The results from these two simulations are shown in Fig.16. Clearly there are no visible differences in these two results. The parameter, $\kappa_{0} \triangle t /(\triangle x)^{2}=10^{4}$, measured by the size of finest rectangular cell $\triangle x$, is so large that the formulation for the material in the middle should be good for steady states. Figure 17 displays the results of the equivalent three dimensional problem.
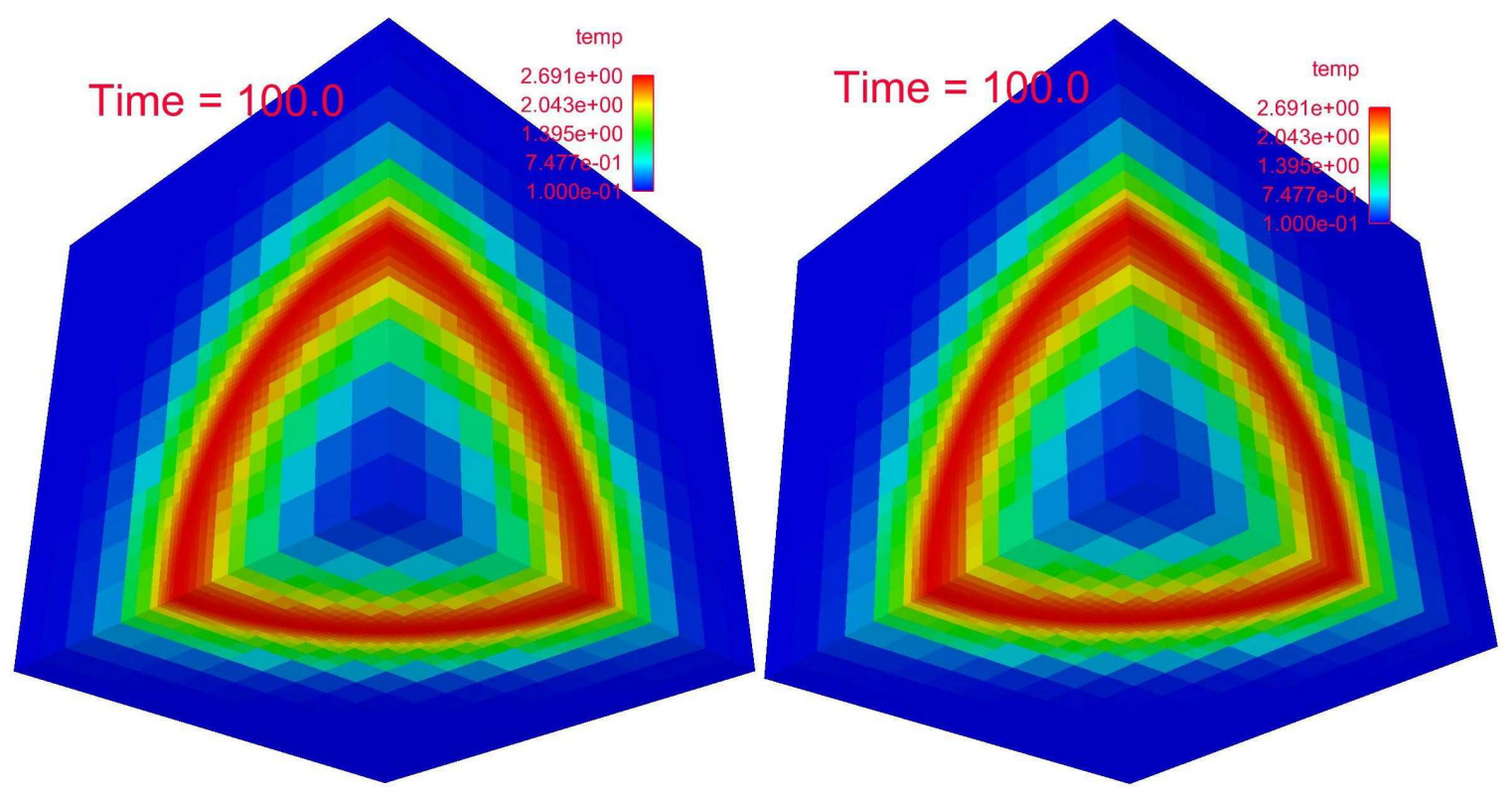

Figure 17: Temperature at $\mathrm{t}=100$ from a three dimensional simulation.with fixed time step $\triangle t=0.001$ (the left image) and $\triangle t=1.0$ (the right image).

In the next example, we examine a problem with ten materials. Figure 18 gives the distrtibution of heat capacity (top left), material diffusion coefficient (top right), radiation diffusion coefficient (bottom left), and initial temperature (bottom right). We give the numerical solutions at $\mathrm{t}=10$ and $t=1000$ in Fig.19. The result of the equivalent three-dimensional problem is given in Fig.20.

At last, we run two problems in which the initial meshes and volume fractions of each material on cells are simulation results obtained from Roxane [34]. The heat capacity and radiation diffusion coefficient are 1.0 and 0.1 uniformally. The material diffusion coefficients of the five materials in the two-dimensional simulation are shown in the left image of Fig.21, vary from 1 to 10000. The initial temperature is 1.0 in the middle material and 0.01 in the other materials. In the other image of Fig.21, we give the temperature at $t=0.1$ after 1000 cycles. We should point out that the volume fractions in this prblem are not perfectly symetric. In a equilvalent three-dimensional calculation, 


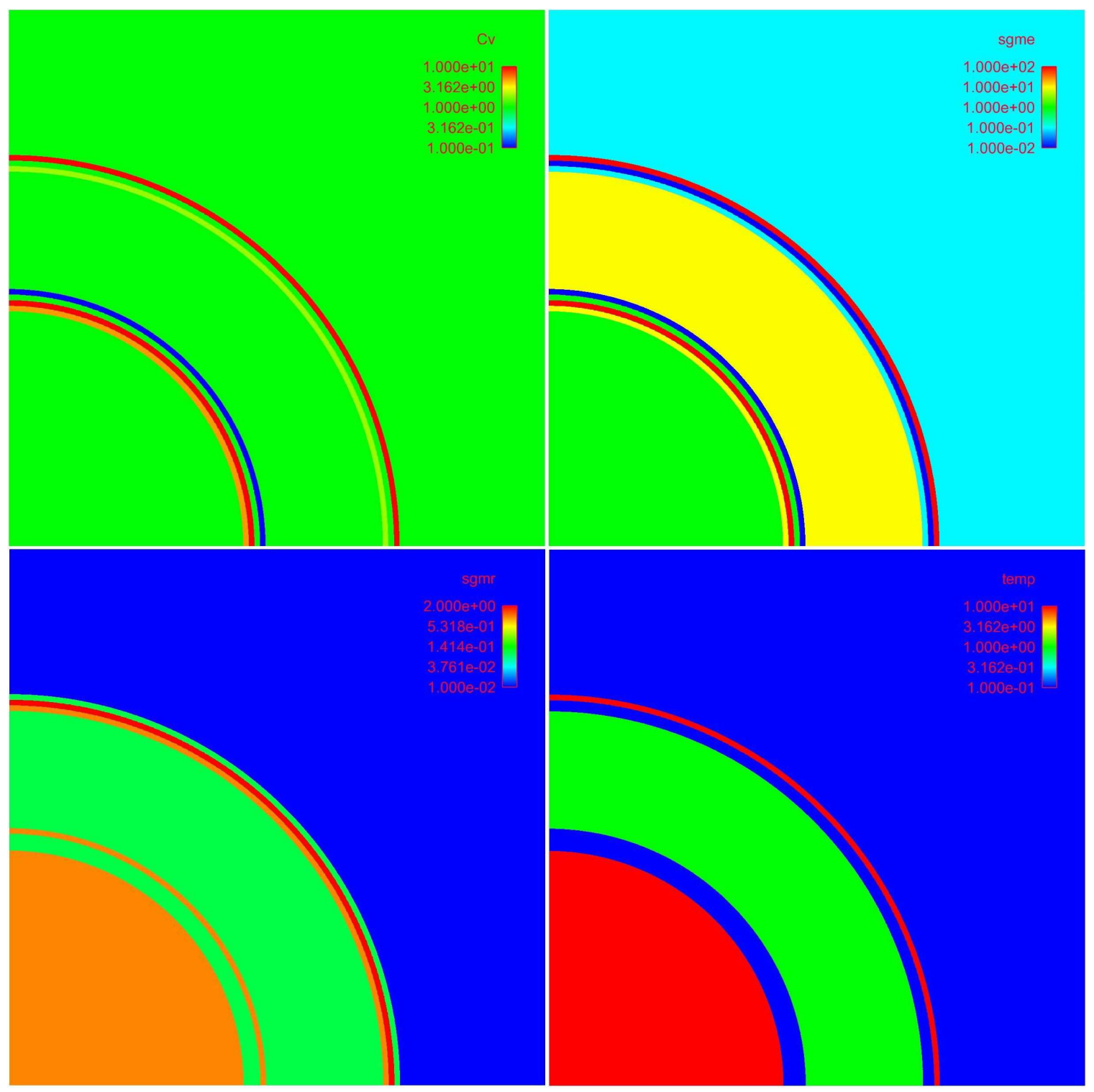

Figure 18: Distrtibution of heat capacity varing from 0.1 to 10 (top left), material diffusion coefficient from 0.01 to 100 (top right), radiation diffusion coefficient from 0.01 to 2 (bottom left), and initial temperature (bottom right) from 0.1 to 10.0. 

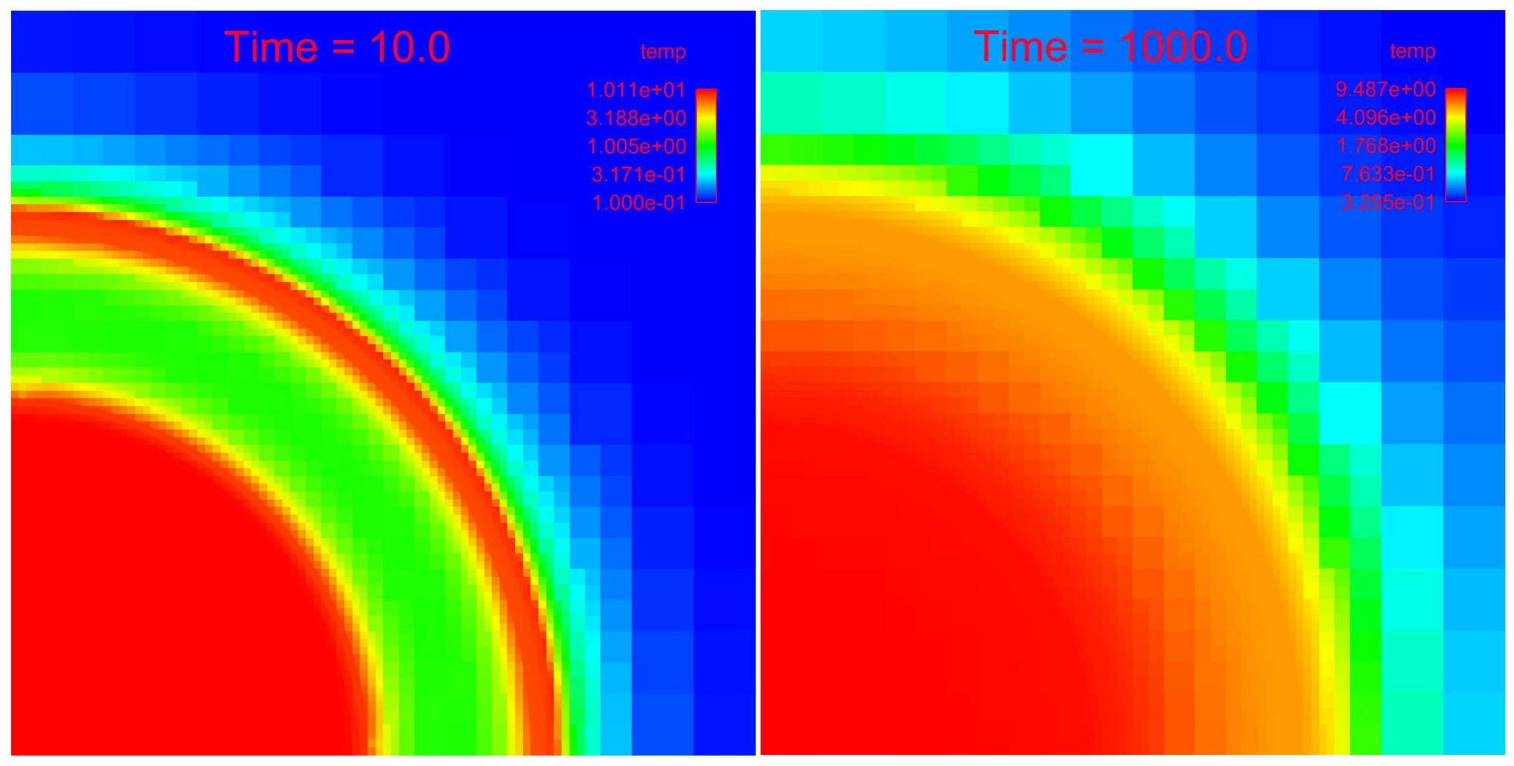

Figure 19: Numerical solutions at $t=10.0$ (the left) and $t=1000.0$ (the right) from a two dimensional simulation with fixed time step $\Delta t=0.1$.
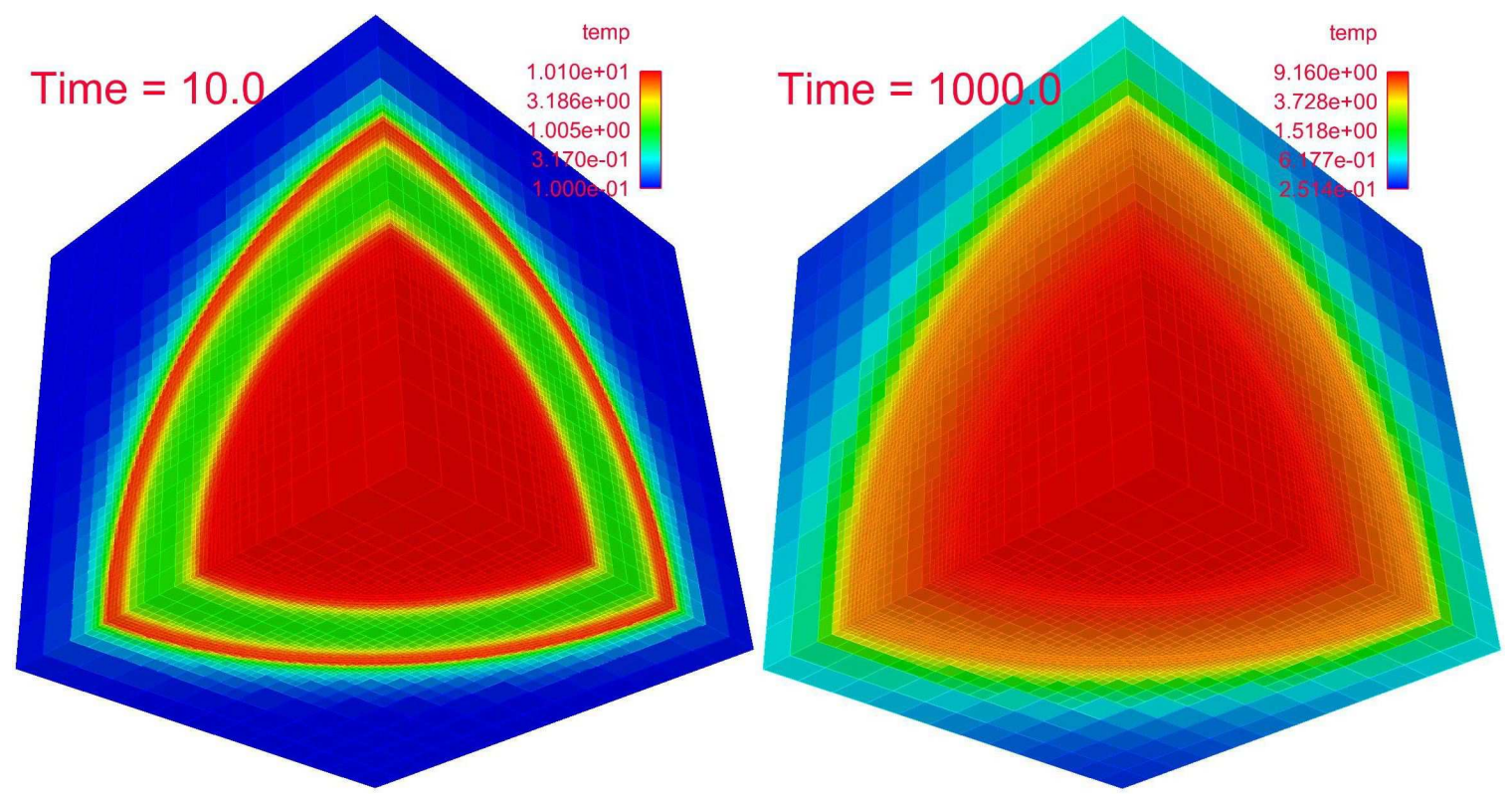

Figure 20: Numerical solutions at $\mathrm{t}=10.0$ (the left) and $\mathrm{t}=1000.0$ (the right) from a three dimensional simulation with fixed time step $\Delta t=0.1$. 


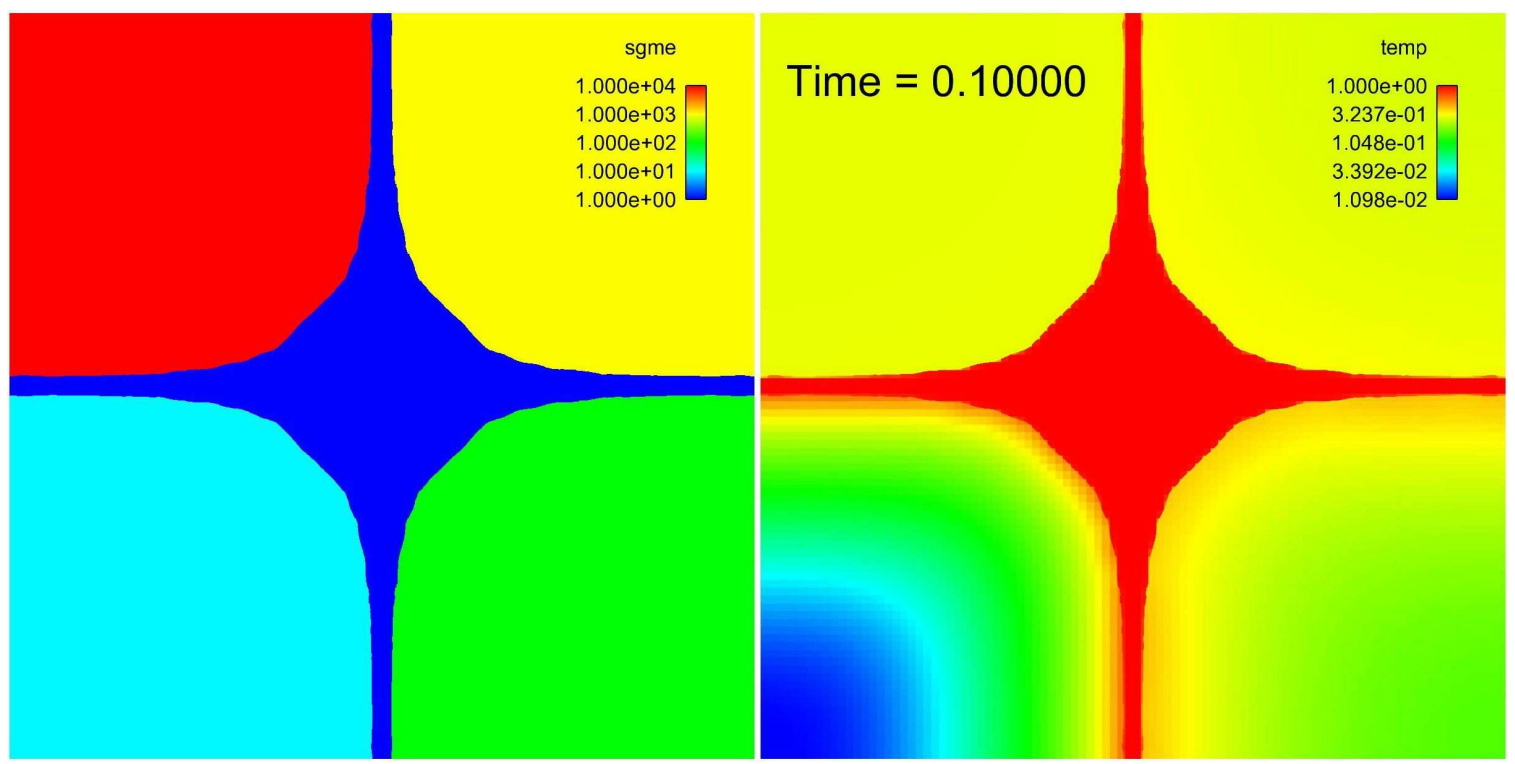

Figure 21: Distribution of material diffusion coefficient and temperature.

the material diffusion coefficients of the material in the central region and the eight corners in the three-dimensional simulations are 1, 10, 100, 1000, 10000, 20, 200, 2000, 20000. The left image of Fig.22 displays the reconstructed material interfaces within mixed cells, and the temperature at time $t=0.1$ is partially shown in the right image of the figure.

\section{Conclusion and Discussion}

Mixed cells are often encountered in numerical simulations, in which there are more than two materials within some cells. To overcome the difficulty of unknown physics property of mixed cells, for example, diffusion coefficients, in numerical simulations for diffusion equations, we have implemented an approach to divide each mixed cell into a set of unstructured cells through linear interface reconstruction in two- and three-dimensions, and then to solve diffusion equations on the reconstructed meshes. The reconstructed meshes include arbitrarily polygons in two dimensions and arbitrarily polyhedrons in three dimensions

We have developed a numerical scheme for diffusion equations on the resulting unstructured meshes. The scheme is based on the conservation of energy for the discontinuity of material property, and the discontinuity is correctly treated in the scheme. To increase the accuracy in time, we have introduced a set of unknowns at the half time step. We have taken special consideration for the scheme to work for large sizes of time step and be able to give correct steady states if the size of time step is very large. The scheme turns to previous methods for structured meshes [11]. We also describe the scheme for meshes with adaptive mesh refinement as a special case. The scheme has been applied to a few test problems to demonstrate its correctness. The features of the scheme about the discontinuity of material property and steady states have been demonstrated in numerical examples.

Interface reconstruction within mixed cells is widely used in numerical simulations for hydrodynamics, called volume of fluid (VOF). As we know, all the practices have used linear interface 

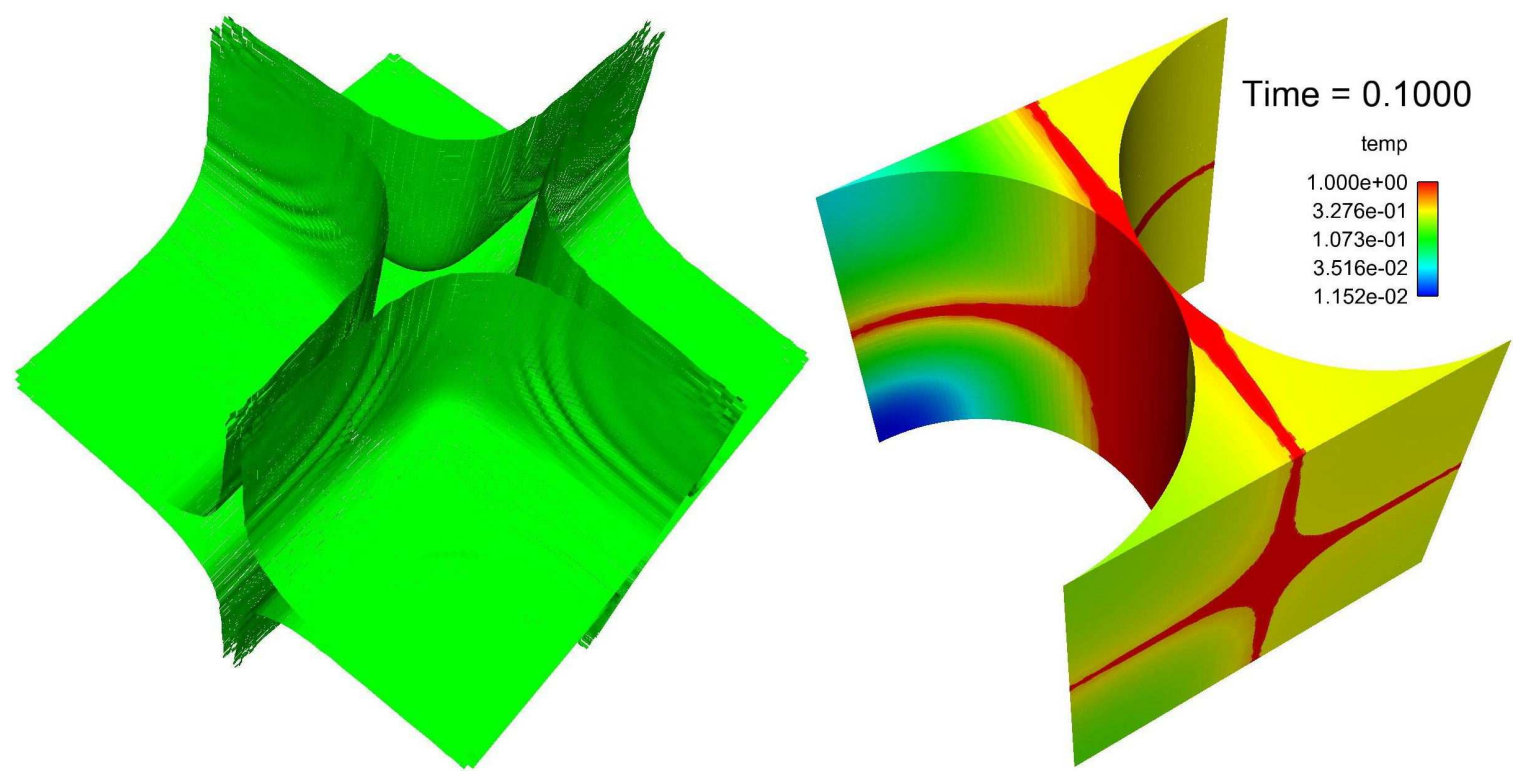

Figure 22: The reconstucted material interfaces (the left) and the numerical solution at $\mathrm{t}=0.1$ after 1000 time steps.

reconstruction, which inevitably results in discontinuous interfaces at cell faces. To eliminate the discontinuity, bilinear reconstruction, through which one interface between two materials within a mixed cell is approximated by two connected line segments. Furthermore, the bilinear segments could be replaced by a curve. But, these techniques, bilinear segment and curve, have never been put into numerical calculations because of the complexity and the concave nature of resulting numerical cells.

The interface between materials is continuous in real physics. The discontinuity resulting linear reconstruction, we believe, like any other approximation, will contribute to overall numerical errors of the scheme. With this error, we still believe the method to be presented in this paper is second order accurate, but we have not proved this. Also, we have not studied the consequence purely due to the discontinuity in numerical simulations. We believe that for unstable physics problems the consequence is more severe in hydrodynamics than in diffusion.

For interface reconstruction, we have used the assumption that all interfaces within a cell are parallel. This assumption is widely used in numerical simulations. Except for the cells at corners at the initial condition of some problems, in which materials are arranged, for example, in a T shape, the assumption is well justified. Even for the cells at the corners, the assumption is acceptable after the sharp corners are smoothed.

We develop this scheme for its use in time-dependent problems in a code of multi-physics simulations, in which existing linear solvers, for example, Hypre, a library developed by Lawrence Livermore National Laboratory, are used to solve linear systems. Nonlinearity is an issue for diffusion solvers. For large time steps, we have to introduce another set of iterations for nonlinearity. It is possible to combine the iteration for implicitness and the one for nonlinearity together so that only a single level of iteration is needed, as demonstrated in [12] for structured meshes. But, if we do this, it will be hard to use existing linear solvers.

It is clear that interface reconstruction contributes additional cost in diffusion solvers. For the 
cost, we run two simulations for a problem with two materials on a two-dimensional uniform mesh with $300 \times 300$ cells for 100 time steps, one with interface reconstruction and the other one with homogenization. In simulation time, the run with the reconstruction takes only $8.2 \%$ more time to finish than the run with homogenization.

As stated before, our multi-physics code is AMR-based, in which diffusion equations are solved on meshes with AMR. This approach has been used in many multi-phsyics codes, for example, $[22,31]$. In our code, we first separate diffusion from other physics, for example, hydrodynamics, through so called operator-splitting technique. In the cell-based AMR, we list the cells on the top level, which do not have children, one by one without ijk-structure. For each cell, before we run any physics, we have already built the connectivity, i.e., neighboring cells of each top cell. The neighboring cells could be at the same level, or only one level high or low, in refinement. This is the constraint we have enforced when the AMR mesh is built for smooth transition of mesh resolution. Within the context of diffusion equation, we track the temperature of each material within a mixed cell since the materials are not necessarily in thermal equilibrium. We divide each mixed cell to a number of sub-cells, as stated in this paper. After that, we calculate the fluxes between any two adjacent cells (or sub-cells) as long as the two has non-zero interface, and form a linear system. The linear system is then solved through a linear solver. Therefore, the diffusion equation is solved globally in our cell-based AMR. We believe that currently the diffusion equation is similarly and globally solved in patch- or block- based AMR. After all the physics packages are updated one time step, we calculate the criterion to refine or coarsen each cell. After that we mark additional cells to be refined for the smooth transition of mesh resolution, and implement mesh refinement and coarsening. Finally, we map the variables from the previous mesh to the new mesh, and start a new time step.

AMR adds a significant computer science burden. But for some of our problems, resolving mixed cells is critical. For that users of our codes typically use six levels of grids in their daily work. Our users just cannot use a uniform mesh at the finest level in general. Our multi-physics code is intrinsically based on AMR. To illustrate the benefit of AMR, we have run two simulations on a domain $1.28 \times 1.28$, one with AMR and the other with a uniform mesh. The mesh with AMR has five levels. The size of the coarsest cell is $0.04 \times 0.04$, and the size of finest cells is $0.0025 \times 0.0025$. The run with a uniform mesh has $512 \times 512$ cells of size $0.0025 \times 0.0025$. In simulation time, the run with the uniform mesh costs 12.3 times of the run with AMR. Figure 23 shows the results at one instant, in which the top two images are from the simulation with AMR, and the bottom two from the one with the uniform mesh.

If our code is only for diffusion, it is unnecessary to use AMR. Since the solver developed in this paper is for its use in multi-physics simulations including shocks and material interface, which require AMR to resolve, the mesh used in this paper is with AMR. AMR helps resolve material interfaces. In this paper, we have not discuss the procedure to refine and coarsen cells. Therefore, the mesh is given and fixed. How to implement an effective AMR is beyond the scope of this paper, but for the completeness of the discussion, we include Fig.24 for the effectiveness of our current AMR code. The left image in the figure is the initial mesh with material interface bwteen two materials, and the image at the right is the mesh after 450 time steps. As shown in the images, mixed cells are always refined at the finest level.

Finally, we would like to mention the parallel efficiency of our code. In our code, we try to combine parallel communications needed for different physics packages into fewer communications. Therefore, our code currently requires five ghost cells beyond the domain of each computer pro- 

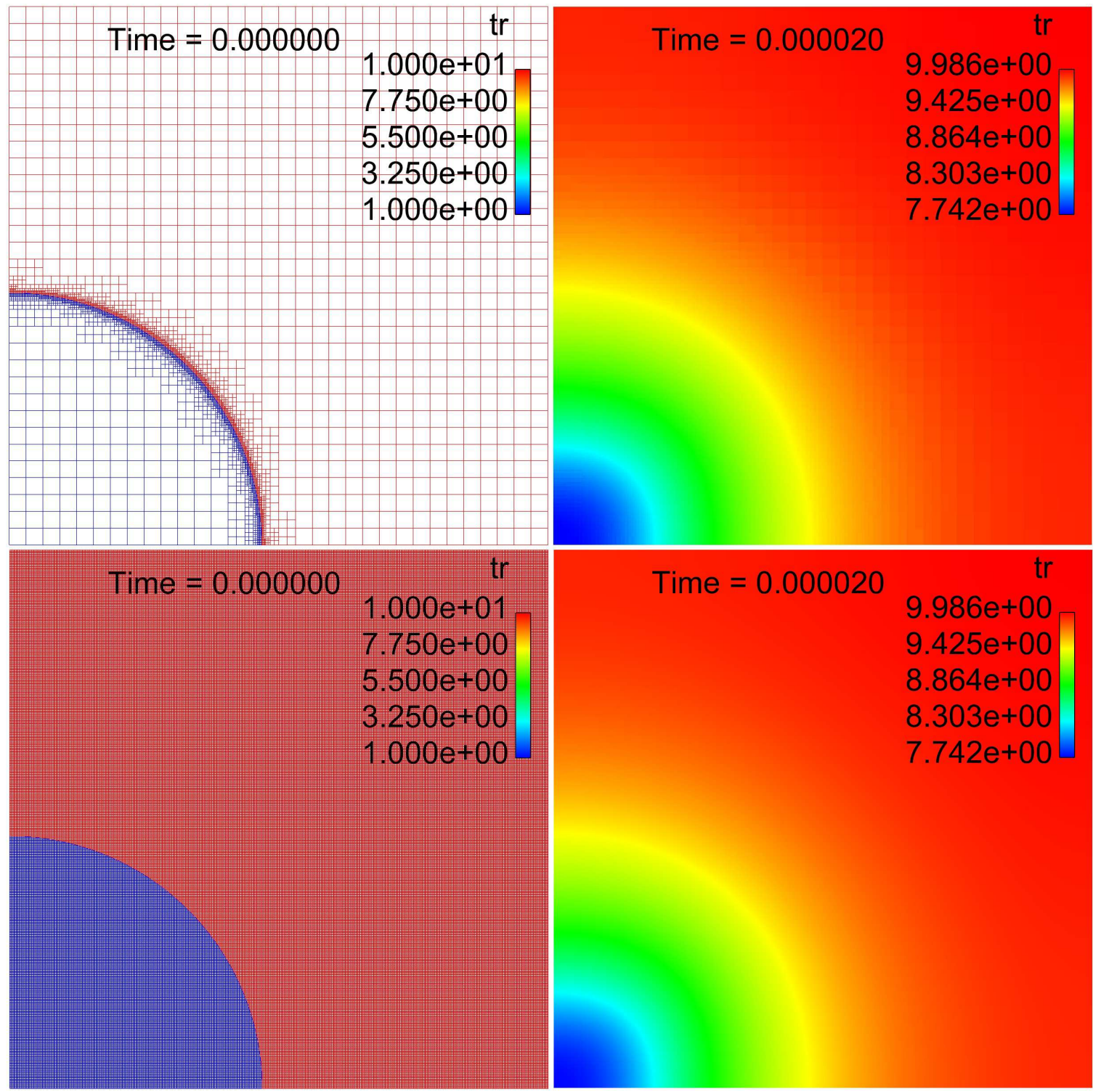

Figure 23: The comparison in mesh and solution between AMR and uniform mesh. The run with AMR has five levels of cells (top two images). The run with uniform mesh has the cells with the same size as the finest cells in AMR (bottom two images). 

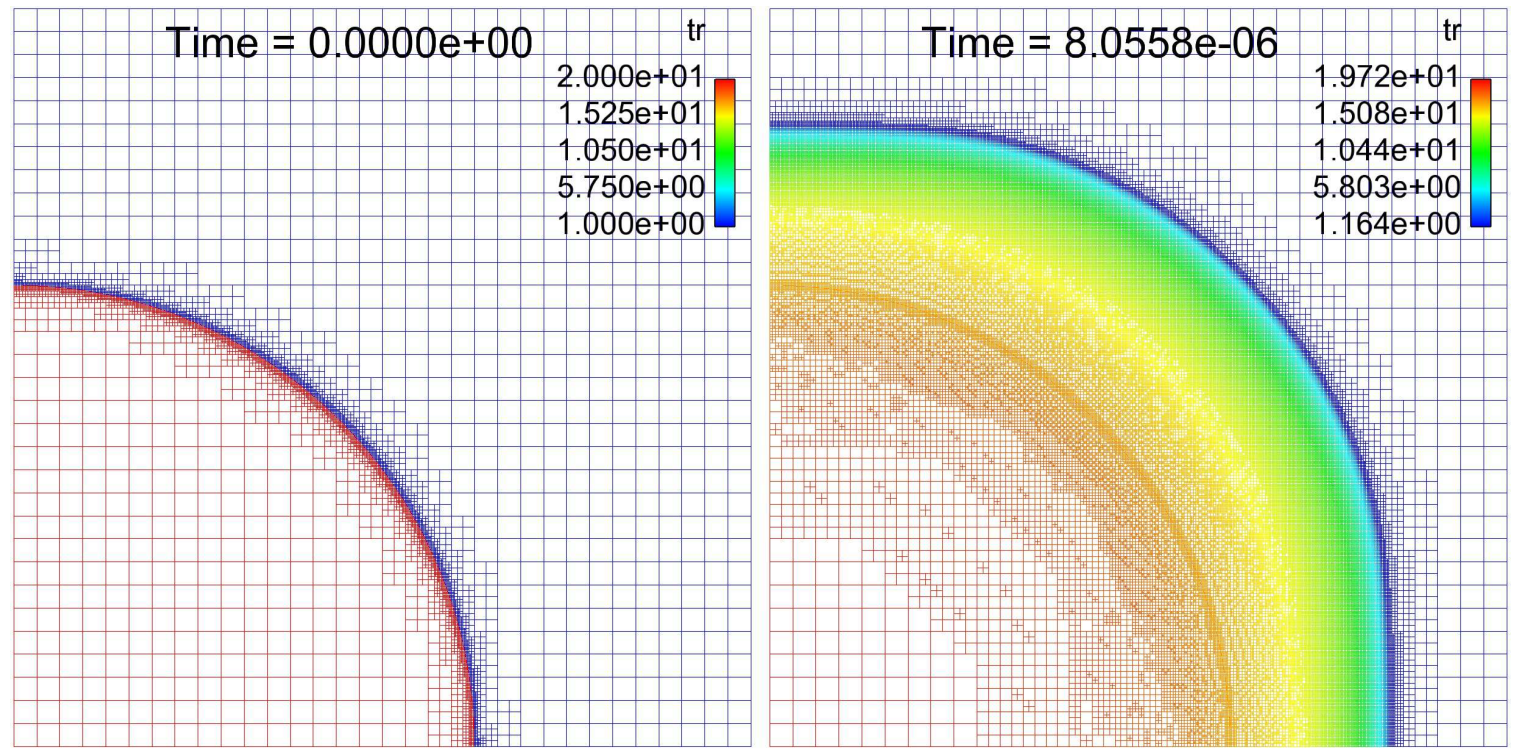

Figure 24: Meshes at the initial time and after 450 time steps.

cessor. This is illustrated in Fig.25, in which the cells with blue color are the domain of one processor, while the cells with red color beyond the domain are ghost cells of this processor. The ghost cells are built before any physics package starts calculation and after the whole simulation domain is repartitioned among a given number of processors. These ghost cells result in redundant calculations compared with the run on a single processor.

To get a rough idea of parallel efficiency, we have implemented several calculations with different numbers of processors for a same problem with our multi-material scheme on a mesh with five levels of AMR. The simulation domain is $1.28 \times 1.28$, and coarsest and finest cells have their sizes $0.01 \times 0.01$ and $0.000625 \times 0.000625$ respectively. The plot in Fig. 6 shows the parallel efficiency in which the vertical axis is the ratio of the time used in parallel runs divided by the time needed in the run of a single processor.

\section{Acknowledgments}

This research and development are funded by the United States Department of Energy ASC program. The authors are grateful to Frederick L. Cochran and Chong Chang for discussions about this work.

\section{References}

[1] S.V. Patankar, Numerical Heat Transfer and Fluid Flow, Hemisphere Publishing Corp., New York, 1980

[2] M.A. Hogge, A comparison of two- and three-level integration schemes for non-linear heat conduction, in Numerical Methods in Heat Transfer, Eds. R.W. Lewis, K. Morgan and O.C. Zienkiewicz, John Wiley, New York, pp.75-90, 1981. 


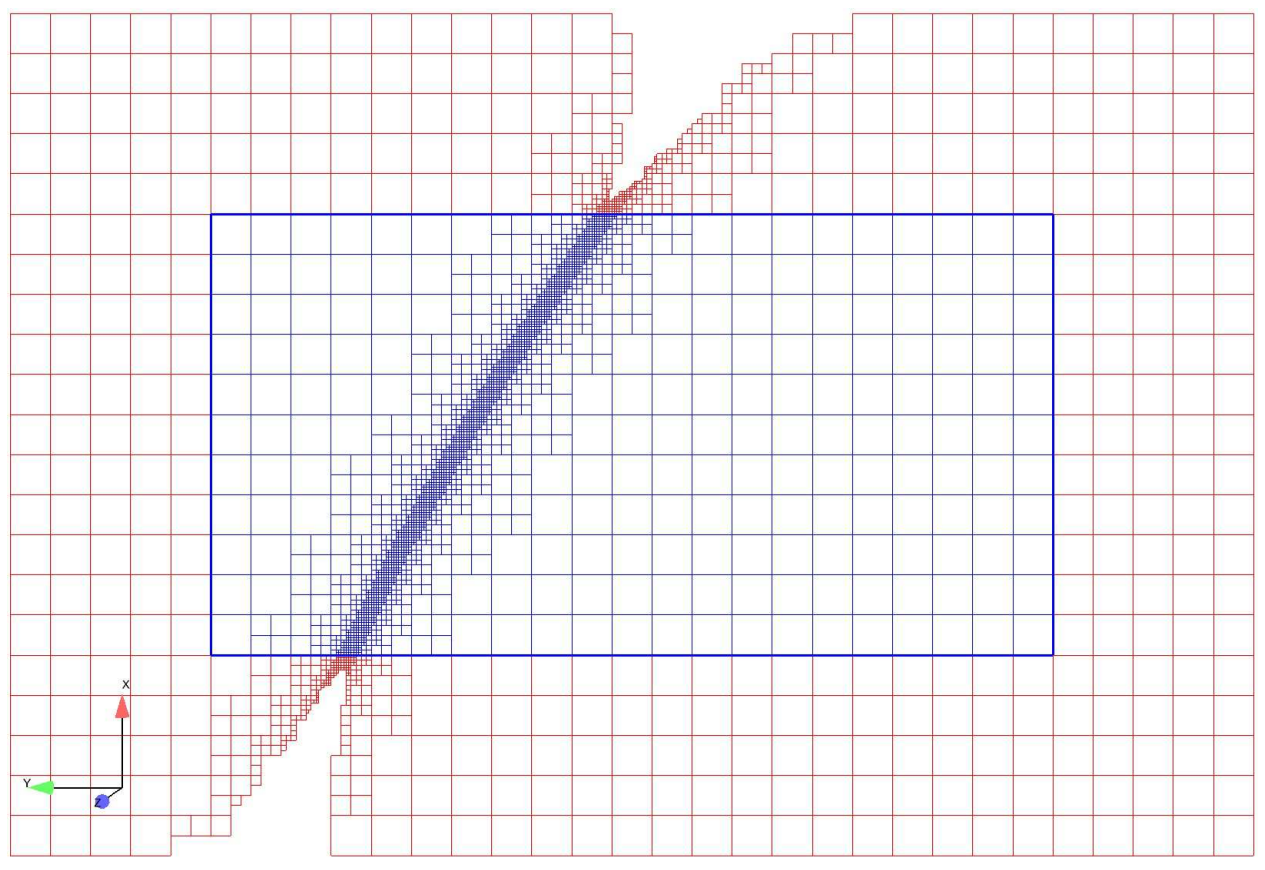

Figure 25: The domain of one computer processor (blue cells) and the ghost cells of this processor (red cells).

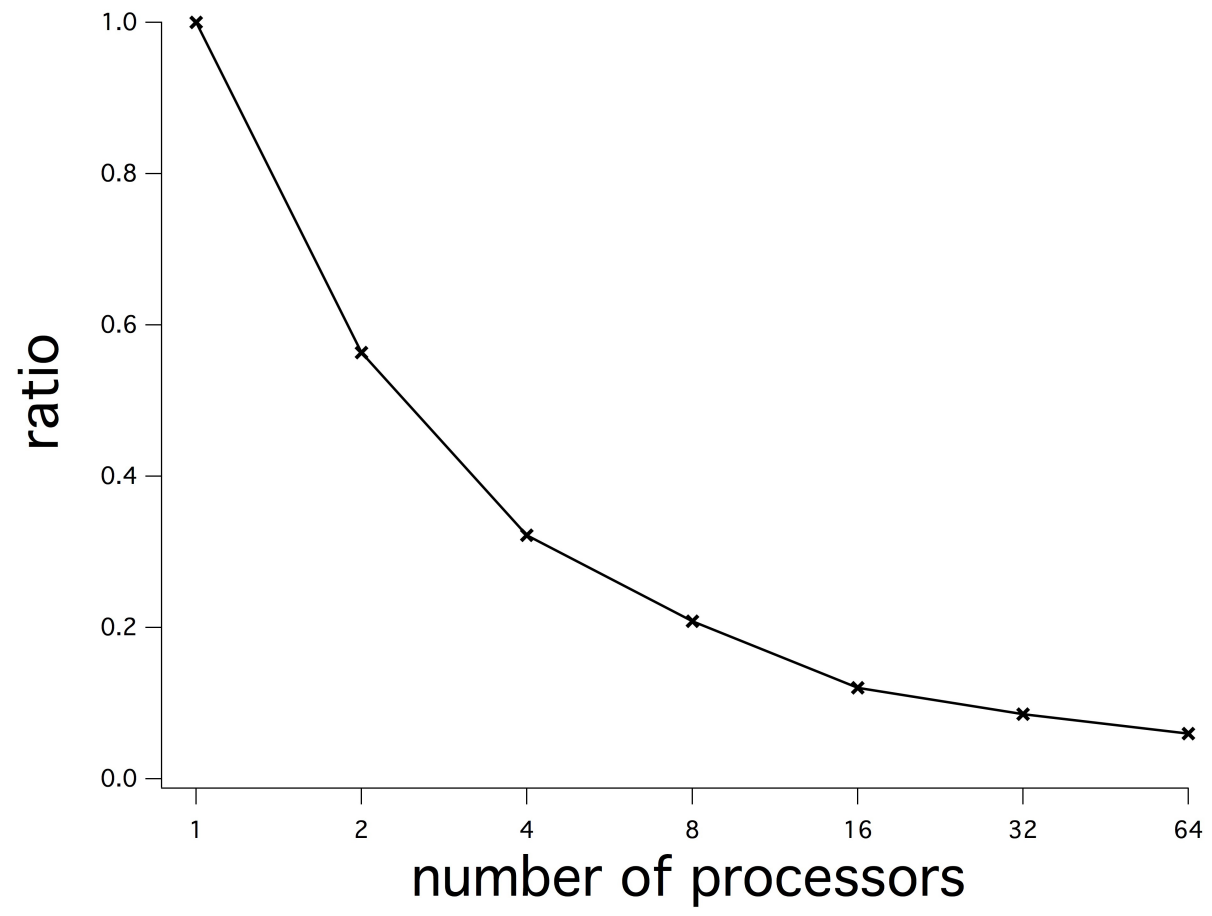

Figure 26: Performance of parallel runs for a problem with fixed size. The vertical axis is the time of parallel runs divided by the time needed for the run of a single processor. 
[3] M. Jakob, Heat Transfer, Vol. 1, Wiley, New York, 1949.

[4] R.E. Alcouffe, A. Brandt, E. Dendy, and J. Painter, the multigrid method for diffusion equation with strong discontinuous coefficients, SIAM J. Sci. Stat. Comput., 2, 430, 1981.

[5] B.A. Fryxell, P.R. Woodward, P. Colella, and K.-H. Winkler, An implicit-explicit hybrid method for Lagrangian hydrodynamics, J. Comput. Phys., 63, 283, 1986.

[6] Y. Jaluria and K. E. Torrance, Computational Heat Transfer (Spring-Verlag, Berlin, 1986).

[7] W. Dai and P. R. Woodward, Iterative implementation of an implicit-explicit hybrid scheme for hydrodynamics, J. of Comput. Phys., 124, pp.217-229, 1996.

[8] M. Shashkov and S. Steinberg, Solving diffusion equations with rough coefficients in rough grids, J. Comput. Phys.,129, 383-405, 1996.

[9] J. Hyman, M. Shashkov, and S. Steinberg, The Numerical Solution of Diffusion Problems in Strongly Heterogeneous Non-isotropic Materials, J. Comput. Phys., 132, pp.130-148, 1997.

[10] W. J. Rider and D. B. Kothe, Reconstructing volume tracking, J. Comput. Phys.,141, 112-152, 1998.

[11] W. Dai and P.R. Woodward, Numerical Simulations for Nonlinear Heat Transfer in a System of Multimaterials, J. Comput. Phys., 139, pp.58-78, 1998.

[12] J. Morel, R. Roberts, and M. Shashkov, A local support-operators diffusion discretization scheme for quadrilateral r z meshes. J. Comput. Phys., 144, pp.1751, 1998.

[13] W. J. Rider, D. A. Knoll, and G. L. Olson, A Multigrid NewtonKrylov Method for Multimaterial Equilibrium Radiation Diffusion, J. Comput. Phys., 152, pp.164-191 (1999).

[14] D. A. Knoll, W. J. Rider, and G. L. Olson, An efficient nonlinear solution method for nonequilibrium radiation diffusion, J. of Quantitative Spectroscopy and Radiative Transfer, vol. 63 (1), pp.15-29 (1999).

[15] V. A. Mousseau, D. A. Knoll, and W. J. Rider, Physics-based preconditioning and the NewtonKrylov method for non-equilibrium radiation diffusion, J. Comput. Phys., 160 (2), pp.743765 (2000).

[16] W. Dai and P. R. Woodward, Numerical simulations for radiation hydrodynamics II. transport limit, J. Comput. Phys., 157, pp.199-233, 2000.

[17] T. Palmer, M. Zika, and N. Madsen. Unstructured polyhedral mesh thermal radiation diffusion. Transactions of the American Nuclear Society, 83, pp.2489, 2000.

[18] D. J. Mavriplis, Multigrid approaches to non-linear diffusion problems on unstructured mesh, Numer. Linear Algebra Appl., vol.8, pp.499-512 (2001)

[19] D.A. Knoll, W.J. Rider, G.L. Olson, Nonlinear convergence, accuracy and time step control in non-equilibrium radiation diffusion, Journal of Quantitative Spectroscopy and Radiative Transfer, 65, pp. 2536 (2001).

[20] D. J. Mavriplis, An assessment of linear versus nonlinear multigrid methods for unstructured mesh solvers, J. Comput. Phys., 175 (1), pp.302-325 (2002). 
[21] J. Hyman, J. Morel, M. Shashkov, and S. Steinberg, Mimetic finite difference methods for diffusion equations, Comp. Geosciences, 6(3-4), pp.333-352, 2002.

[22] L. H. Howell and J. A. Greenough, Radiation diffusion for multi-fluid Eulerian hydrodynamics with adaptive mesh refinement, J. Comput. Phys., 184, pp.53-78 (2003).

[23] J. E. Pillod and E. G. Puckett, Second-order accurate volume-of-fluid algorithms for tracking material interfaces, J. Comput. Phys., 199, pp.465-502, 2004.

[24] K. Lipnikov, J. Morel, and M. Shashkov, Mimetic finite difference methods for diffusion equations on non-orthogonal non-conformal meshes, J. Comput. Phys., 199, pp.589-597, 2004.

[25] D. L. Ropp, J. N. Shadid, and C. C. Ober, Studies of the Accuracy of Time Integration Methods for Reaction-Diffusion Equations, J. Comput. Phys., 194 (2), pp.544-574 (2005).

[26] V. Dyadechko and M. Shashkov, Moment-of-fluid interface reconstruction, Technical Report LA-UR-05-7571, Los Alamos National Laboratory (2005).

[27] M. Pernice and B. Philip, Solution of Equilibrium Radiation Diffusion Problems Using Implicit Adaptive Mesh Refinement, SIAM Journal on Scientific Computing, 27 (5), pp.1709-1726 (2006).

[28] G. L. Olson, Efficient solution of multi-dimensional flux-limited nonequilibrium radiation diffusion coupled to material conduction with second-order time discretization, J. Comput. Phys., 226 (1), pp.1181-1195 (2007).

[29] H. T. Ahn and M. Shashkov, Multi-material interface reconstruction on generalized polyhedral meshes, J. Comput. Phys., 226, 2096-2132 (2007).

[30] M. L. Witeka, J. Teixeira, and P. J. Flataud, On stable and explicit numerical methods for the advectiondiffusion equation, Mathematics and Computers in Simulation, 79 (3), pp. 561570 (2008).

[31] M. Gittings, R. Weaver, M. Clover, T. Betlach, N. Byrne, R. Coker, E. Dendy, R. Hueckstaedt, K. New, W. Oakes, D. Ranta, R. Stefan, Computational Science \& Discovery 1 (2008).

[32] J. Yue, G. Yuan, and Z. Sheng, Picard-Newton Iterative Method for Multimaterial Nonequilibrium Radiation Diffusion Problem on Distorted Quadrilateral Meshes, Proceedings of the World Congress on Engineering 2009 Vol II WCE 2009, July 1 - 3, 2009, London, U.K.

[33] A. S. Dawes, Sub-zonal models for the diffusion equations with multi-material mixed cells, Compters \& Fluids, 46, 197-200 (2011).

[34] A.J. Scannapieco, F. Cochran, J. Painter, C. Chang, W. Dai, Roxane: A code for sumulations of multi-physics, Technical Report LA-UR-12-23138, Los Alamos National Laboratory (2012).

[35] T. Feng, H. An, X. Yu, Q. Li, and R. Zhang, On linearization and preconditioning for radiation diffusion coupled to material thermal conduction equations, J. Comput. Phys., 236 (1), pp.2840 (2013).

[36] A. Dawes, Multi-material polygon based finite volume method for diffusion, presentation in 2013 Multi-material Hydrodynamics Conference, San Francisco, CA, September 2-6, 2013;

https://multimat13.1lnl.gov/media/pdf/papers/MultiMat_2003_Dawes.pdf . 
[37] R. M. J. Kramer, and S. J. Mosso, Automatic ordering for volume-of-fluid interface reconstruction in multi-material elements, presentation in 2013 Multi-material Hydrodynamics Conference, San Francisco, CA, September 2-6, 2013;

https://multimat13.1lnl.gov/media/pdf/papers/MultiMat_2003_Kramer.pdf . 